\title{
Effect of perforation on flow past a conic cylinder at $R e=100$ : wavy vortex and sign laws
}

\author{
L. M. $\operatorname{Lin}^{1} \cdot$ X. F. Zhong ${ }^{1} \cdot$ Y. X. Wu $\mathbf{W u}^{1,2}$
}

Received: 27 May 2017 / Revised: 9 October 2017 / Accepted: 8 January 2018 / Published online: 2 April 2018

(c) The Chinese Society of Theoretical and Applied Mechanics; Institute of Mechanics, Chinese Academy of Sciences and Springer-Verlag GmbH Germany, part of Springer Nature 2018

\begin{abstract}
In order to find the intrinsic physical mechanism of the original Kármán vortex wavily distorted across the span due to the introduction of three-dimensional (3-D) geometric disturbances, a flow past a peak-perforated conic shroud is numerically simulated at a Reynolds number of 100. Based on previous work by Meiburg and Lasheras (1988), the streamwise and vertical interactions with spanwise vortices are introduced and analyzed. Then vortex-shedding patterns in the near wake for different flow regimes are reinspected and illustrated from the view of these two interactions. Generally, in regime I, spanwise vortices are a little distorted due to the weak interaction. Then in regime II, spanwise vortices, even though curved obviously, are still shed synchronously with moderate streamwise and vertical interactions. But in regime III, violently wavy spanwise vortices in some vortex-shedding patterns, typically an $\Omega$-type vortex, are mainly attributed to the strong vertical interactions, while other cases, such as multiple vortex-shedding patterns in sub-regime III-D, are resulted from complex streamwise and vertical interactions. A special phenomenon, spacial distribution of streamwise and vertical components of vorticity with specific signs in the near wake, is analyzed based on two models of streamwise and vertical vortices in explaining physical reasons of top and bottom shear layers wavily varied across the span. Then these two models and above two interactions are unified. Finally two sign laws are summarized: the first sign law for streamwise and vertical components of vorticity is positive in the upper shear layer, but negative in the lower shear layer, while the second sign law for three vorticity components is always negative in the wake.
\end{abstract}

Keywords Vortex-shedding pattern $\cdot$ Flow control $\cdot$ Vortex dynamics $\cdot$ Conic disturbance $\cdot$ Perforation $\cdot$ Sign law

\section{Introduction}

Bluff bodies are commonly used in many engineering applications, for instance, mooring cables, flexible risers, and pipelines between oil platforms and submarine drilling wells, cables in suspension bridges, and heat exchangers. Viscous flow leads to vortices generated and alternatively to shedding behind the bluff body at higher Reynolds numbers. Meanwhile, oscillated fluid forces acting on the body also result in the structural vibration, called vortex-induced vibration (VIV). Particularly, as the vortex-shedding frequency

$\triangle$ L. M. Lin

1lmbirthday@163.com

1 Key Laboratory for Mechanics in Fluid Solid Coupling Systems, Institute of Mechanics, Chinese Academy of Sciences, Beijing 100190, China

2 School of Engineering Sciences, University of Chinese Academy of Sciences, Beijing 100049, China is close to the natural frequency of the structure, the sudden amplification in structural amplitude, as well as fluid forces, results in structural fatigue failure and break of structural integrity. Therefore, many experiments and numerical simulations have been carried out in investigating and understanding the dynamics of VIV in recent decades (see in comprehensive reviews of Sarpkaya [1], Williamson and Govardhan [2,3]). Correspondingly, in order to suppress VIV, many methods have been proposed over the last half century and are mainly attributed to controlling the wake dynamics and alleviating the unsteady fluid loadings (see in review works of Sarpkaya and Isaacson [4] and Kumar et al. [5]). For example, there are surface bumps [6], rotating cylinders in the body's boundary layer [7], ventilated trousers [8], traveling wave walls [9], streamline fairings [10], splitter plates [11], helical strakes [12], wavy stagnation surfaces [13,14], radial disturbances on cylinder surfaces $[15,16]$, and peak perforations [17]. 
Before introducing these methods in the flow past the bluff body, typically circular cylinders, a stable laminar vortex shedding regime is in the range of $R e=40-150$ and a transition regime of $R e=150-300$ [18]. Beyond the critical Reynolds number for the onset of the wake instability near $R e_{c}=49$, the flow is unsteady and vortex shedding occurs in the near wake. The first three-dimensional (3-D) transition in the wake of the circular cylinder occurs at $R e \approx 194$, referred to as mode A [19]. Mode A appears as a waviness of the spanwise vortices with a wavelength of around 3-4 diameters and is characterized by the formation of vortex loops that connect two consecutive spanwise Kármán vortices of opposite signs. As the Reynolds number is further increased up to 230-250, another type of 3-D instability known as mode B is appeared and characterized by fine-scale streamwise vortices with a small wavelength of around one diameter. Except for these two small-scale structures in the transition regime, it is found that large scale structures, spot-like vortex dislocations, are generated between spanwise cells of different frequency when the primary vortices move out of phase with each other, at the sites of particular vortex loops, typical of mode A instability, and evolve spontaneously along the span. Therefore, it is clear that the flow past the straight cylinder at $R e=100$ is typically two-dimensional (2-D) and stable to 3-D perturbations. Correspondingly, the shear layers on the top and bottom surfaces and spanwise vortex shedding are always 2-D or non-wavy across the span, as well as the wake width.

However, the introduction of VIV suppression methods would interfere with the original Kármán vortex shedding. Typically, at a certain wave steepness (the ratio of the wave height to the wavelength of wavy disturbance) for a wholly wavy circular cylinder, no evidence of regular Kármán vortex shedding is observed [13]. Similarly, the phenomenon of completely suppressing Kármán vortex shedding can also be achieved by introducing control surface bumps [6], a traveling wave wall [9], wholly wavy stagnation surfaces for square cylinder [14,20], and harmonic and conic disturbances for circular cylinder $[15,16]$. In addition, at lower Reynolds numbers, more complex vortex-shedding patterns are obtained by the introduction of geometric disturbances, such as the large unsteady hairpin structures [14] or $\Omega$-type vortices, "cloud"-like vortices, obliquely shedding vortex, and crossed spanwise vortices with opposite signs [21]. Moreover, these introduced 3-D geometrical perturbations lead not only to spanwise vortices, but also to shear layers wavily varied along the spanwise direction, and a periodic variation of the wake width across the span is observed behind the body.

Additionally, streamwise and vertical vortices appear and are shed, accompanied by the shedding spanwise vortices, due to the introduced geometric disturbances. These additional components of vorticity are firstly generated on 3-D disturbed surfaces with specific distributions and signs [16, 20], as a direct result of introduced geometric disturbances. After these vorticies are convected and transported into the near wake, there is evidence of streamwise and vertical vortices in the near wake varied in signs in an alternate way across the span $[16,21]$. Their senses of rotation are such as to induce the variation in wake width mentioned previously. Typically, up to now, there are two types of explanation described by the streamwise [14] and vertical vortex pairs [22], respectively. It is still unclear if there are some intrinsic connections between these two explanations.

On the other hand, it is reasonable to believe that at lower Reynolds numbers, such as 100, the streamwise and/or vertical vortices, generated on surfaces by 3-D geometric disturbances, in the near wake interfere with shear layers and shedding spanwise vortices. Then original Kármán vortices are disturbed and wavily varied across the span. Particularly, once the spanwise vortex is distorted violently, specific types of vortex are formed, such as the $\Omega$-type vortex. This has already been studied for the interactions between streamwise and spanwise vortices in a previous basic work by Meiburg and Lasheras [23]. Then it is interesting to ask whether there are the interactions between vertical and spanwise vortices. Furthermore, it seems that there are inner triangle relationships among the mentioned three aspects: spacial distributions of streamwise and vertical vortices with specific signs, wavily varied wake width due to streamwise and vertical vortices, and wavy spanwise vortices because of interactions with streamwise and vertical vortices.

The aim of the current work is to try to find the intrinsic physical mechanisms of the original spanwise vortex wavily distorted by introducing 3-D geometric disturbances. The analysis is mainly carried out in the physical connections among the three aspects mentioned. Numerical simulations of the flow past the peak-perforated conic shrouds at Reynolds number of 100 are performed [21].

This paper is outlined as follows. At first, the numerical simulations, including the physical model, numerical methods, boundary conditions, and control parameters, are presented in Sect. 2. Then in Sect. 3, we present the main results of the effect of introduced peak perforation at conic disturbances on wavy vortex shedding. Two types of interactions with the spanwise vorticies are firstly introduced to illuminate distorted spanwise vortices. Then different vortexshedding patterns in the near wake are analyzed on the basis of the two interactions. The wavy shear layers are discussed with different explanations. The physical phenomenon of the spacial distributions of three components of vorticity with specific signs in the top and bottom shear layers and near wake is illustrated. Thus, two sign laws are obtained. Finally, the conclusions in Sect. 4 are presented in brief. 


\section{Physical model}

As shown in Fig. 1, an incompressible flow with constant density $\rho$ and kinematic viscosity $v$ past two types of circularsection cylinders is taken into account. The inertial Cartesian coordinate system $(x, y, z)$ is then established with the $x$ axis (streamwise) aligned with the incoming flow at velocity $U_{\infty}$, the $z$-axis (spanwise) aligned with the central axis of cylinder, and the $y$-axis (vertical) perpendicular to the $(x, z)$ plane. As shown in Fig. 1a, the conic disturbance as a shroud is installed outside of the straight circular cylinder, referred to as the conic cylinder or shroud (denoted by "C" in figures and their titles). As shown in Fig. 1b, the perforation is introduced at peak of such conic disturbance with several circular holes uniformly distributed along the azimuthal direction, referred to as the peak-perforated conic shroud (denoted by "Co"). The basic conic shroud can be mathematically described by $r(z)=\frac{1}{2} D+\xi$, where $\xi$ is the conic disturbance in the radial $(r)$ and spanwise plane, $\xi(z)=\frac{2 W}{\lambda} z\left(z \in\left[0, \frac{1}{2} \lambda\right]\right)$ and $\xi(z)=2 W\left(1-\frac{z}{\lambda}\right)\left(z \in\left[\frac{1}{2} \lambda, \lambda\right]\right), \lambda$ is the wavelength, $W$ is the peak-to-valley wave height, and $D$ is the base diameter. Correspondingly, the peak and valley are defined as spanwise positions with maximal and minimal diameters, i.e. $(2 W+$ $D)$ and $D$, respectively. In addition, Fig. 1c presents three parameters used to describe the introduced perforation, the diameter of holes $\mathrm{Do}$, their number $\mathrm{No}$, and attack angle $\mathrm{Ag}$ between the central axis of hole near the front stagnation point of cylinder and incoming flow.

The non-dimensional continuity and Navier-Stokes equations are written by

$\nabla \cdot \boldsymbol{u}=0$

$\frac{\partial \boldsymbol{u}}{\partial t}+(\boldsymbol{u} \cdot \nabla) \boldsymbol{u}=-\nabla p+\frac{1}{R e} \nabla^{2} \boldsymbol{u}$,

where $\boldsymbol{u}=(u, v, w)$ is the velocity vector, $p$ is the static pressure, $t$ is the time, $\nabla$ is the gradient operator, and $R e$ is the Reynolds number, $R e=U_{\infty} D / \nu$. Lengths are scaled by the base diameter $D$, velocities by the free-stream velocity $U_{\infty}$, and then time by $D / U_{\infty}$.

In order to obtain the appropriate numerical solutions, boundary conditions should be properly proposed. To simulate flows around cylinders with infinite span, the flow is assumed to be periodic along the spanwise direction. As for boundary conditions in the $(x, y)$ plane, the uniform free-stream velocity $\left(\boldsymbol{u}=\left(U_{\infty}, 0,0\right)\right)$ at inlet, simple non-reflecting outflow $(\partial \boldsymbol{u} / \partial x=0)$ at outlet, free slip $(\partial(u, w) / \partial y=v=0)$ at vertical sides and non-slip boundary condition $(\boldsymbol{u}=0)$ on cylinder surfaces are prescribed. Reference pressure is set to be zero at the center of inlet $y=0$.

In flows past conic shrouds with and without peak perforation, there are six control parameters. The first is the
Reynolds number $R e$, and the other five geometric parameters are used to describe the conic disturbance and perforation. Two independent length parameters, the non-dimensional wavelength $\lambda / D$ and the wave steepness $W / \lambda$, for the conic disturbance are obtained in describing the similarity between different conic cylinders. While for the perforation, there are the non-dimensional diameter of holes $D o / D$, number of holes $N o$, and attack angle $A g$. In the present paper, a series of computational investigations have been carried out with different values, as summarized in Table 1. Among them, only four holes are considered. Due to the symmetry of perforation along the $y$-axis, the maximum of attack angle is less than $360^{\circ} /(2 \mathrm{No})=45^{\circ}$ with $\mathrm{No}=4$. In addition, it should be noted here that only special parameter groups composed of $D o$ and $A g$ are considered for limited computational resources. For instance, only cases at $A g=0^{\circ}$ with varied $D o / D$ and at $D o / D=0.1$ with increasing $A g$ are taken into account.

It is also very important that the proper size of computational domain should be carefully selected to weaken its effect on flow field, fluid forces and vortex shedding frequency as much as possible. Based on previous work of Lin [20], the non-dimensional computational domain in the $(x, y)$ plane is considered as to be $40 \times 20(x \times y)$ due to the present computational resources and costs in time, as shown in Fig. 2. The dimensionless computational length in the $z$-axis is given as one period of conic disturbance, i.e. $\lambda / D$, regardless of the effect of larger-scale spanwise disturbance.

Different from the structural grids in a hexahedral form for the straight or conic cylinder, mesh generation for the peak-perforated conic shroud is more complicated due to the appearance of circular holes at peak of the conic disturbance. Typical mesh distributions in the $(x, y)$ plane are shown in Fig. 2. Far away from the cylinder, the hexahedral grids are also adopted and coarsened (it should be explained here that in Fig. $2 b$ those unlike hexahedral grids upstream are actually the result of intersections of a sectioned plane $z=\frac{1}{2} \frac{\lambda}{D}$ and oblique hexahedral grids). Meanwhile, in the flow region near the cylinder, where the distance of the regional boundary away from the conic shroud is $2 D$ in the $x$ and $y$ directions, and holes, the unstructured grids in forms of triangle on the surface and tetrahedron in space are applied and concentrated. Based on previous work of Lin [20], as shown in Fig. 2c, d, the finest non-dimensional grid spacing is on the order of $O\left(10^{-2}\right)$ on the front stagnation surface and $O\left(10^{-3}\right)$ on the rear surface. Moreover, details of numerical simulations could be found out in Refs. [16,17,21], such as total number of grids and verifications for present numerical methods. Here we just present computational results in flows past conic shrouds with and without peak perforations. 

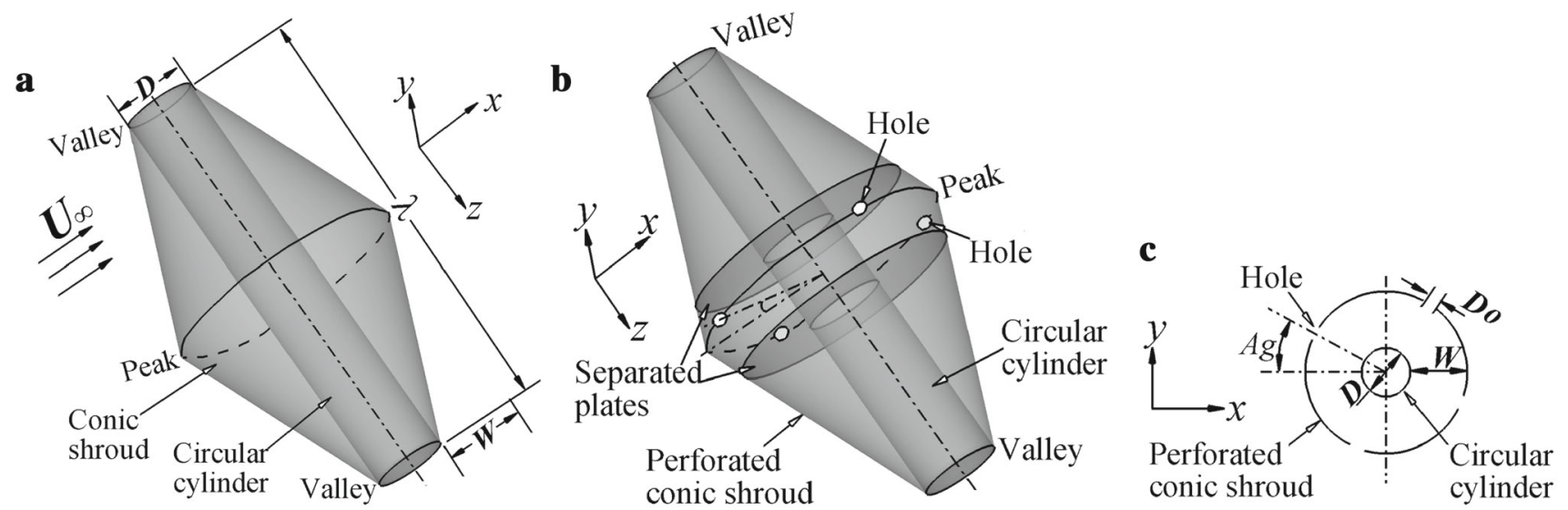

Fig. 1 Schematics of flows past $\mathbf{a}$ a conic shroud and $\mathbf{b}$ a peak-perforated conic shroud with a specific attack angle $\mathrm{Ag}$ in $\mathbf{c}$ the cross-section of peak

Table 1 Summarized of computational parameters with different values

\begin{tabular}{lllllll}
\hline Items & $R e$ & $\lambda / D$ & $W / D$ & $D o / D$ & $A g$ & $N o$ \\
\hline Values & $(100)$ & $(4,6,8)$ & $(0.0125,0.025,0.05,0.1,0.2)$ & $(0.1,0.2,0.4)$ & $\left(0^{\circ}, 15^{\circ}, 30^{\circ}, 45^{\circ}\right)$ & $(4)$ \\
\hline
\end{tabular}
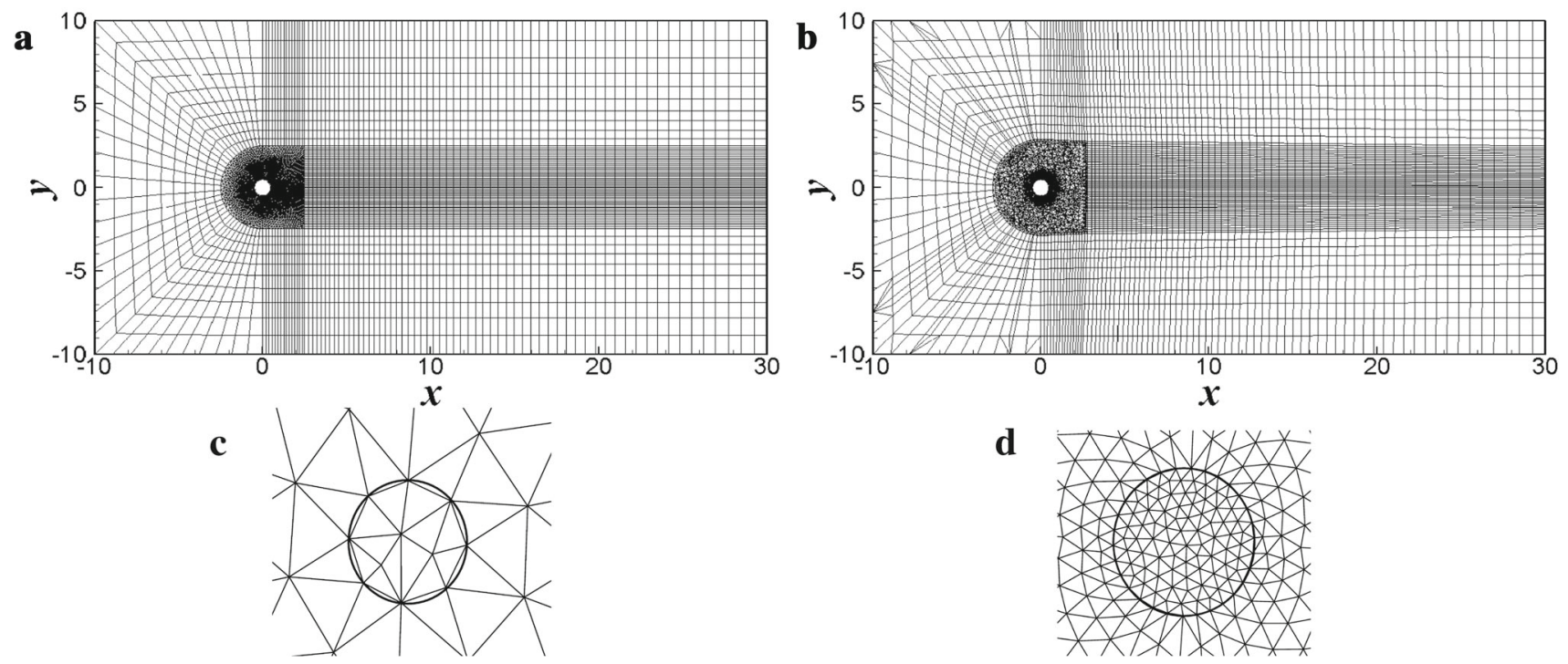

Fig. 2 Non-dimensional computational domain and typical grid distributions at $\mathbf{a} z=0$ and $\mathbf{b} z=\frac{1}{2} \lambda / D$, and mesh for holes (circle line) near $\mathbf{c}$ front and $\mathbf{d}$ rear surfaces

\section{Results and discussion}

\subsection{Flow past the 2-D circular cylinder}

A 2-D simulation was performed past the circular cylinder at $R e=100$ as for the basic case. As shown in Fig. 3a, the wake consists of strong spanwise vortices alternatively shedding from the top and bottom shear layers, identifying the well-known Kármán vortex street. The time histories of drag and lift forces strongly show the purely periodic vortex shedding over the whole computational time, as shown in
Fig. 3b. The values of the global flow coefficients are: the mean drag coefficient $C_{\mathrm{D}_{-} \mathrm{M}}=1.39$, the root-mean-square (RMS) lift coefficient $C_{\mathrm{L} \_} \mathrm{RMS}=0.256$, and the Strouhal number $S t=0.165$. They are in good agreement with previous experiments (Exp.) and numerical simulations (Num.) in Refs. [4,24,25]: $C_{\mathrm{D} \_\mathrm{M}}=1.25-1.4$ or 1.8 and $S t=0.164$ (Exp.); $C_{\mathrm{D} \_\mathrm{M}}=1.37, C_{\mathrm{L} \_} \mathrm{RMS}=0.24$ (evaluated from the maximum $C_{\mathrm{L}}$ ) and $S t=0.167$ (Num.), respectively. By the way, the peak amplitudes of drag and lift coefficients are all constant of 0.01 and 0.36 , respectively. 
$\mathbf{a}$

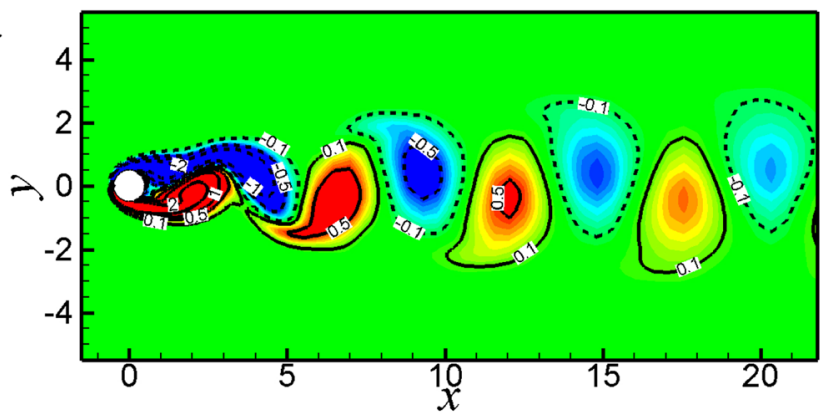

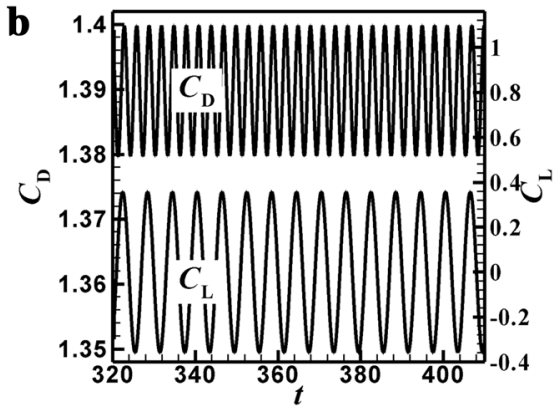

Fig. 3 a Contours of the spanwise vorticity in the basic case describing the Kármán vortex shedding at $R e=100$ and $\mathbf{b}$ time histories of drag and lift coefficients, $C_{\mathrm{D}}$ and $C_{\mathrm{L}}$, where the red and blue contours, as well as the solid and dashed lines, denote the positive and negative values of spanwise vorticity, respectively
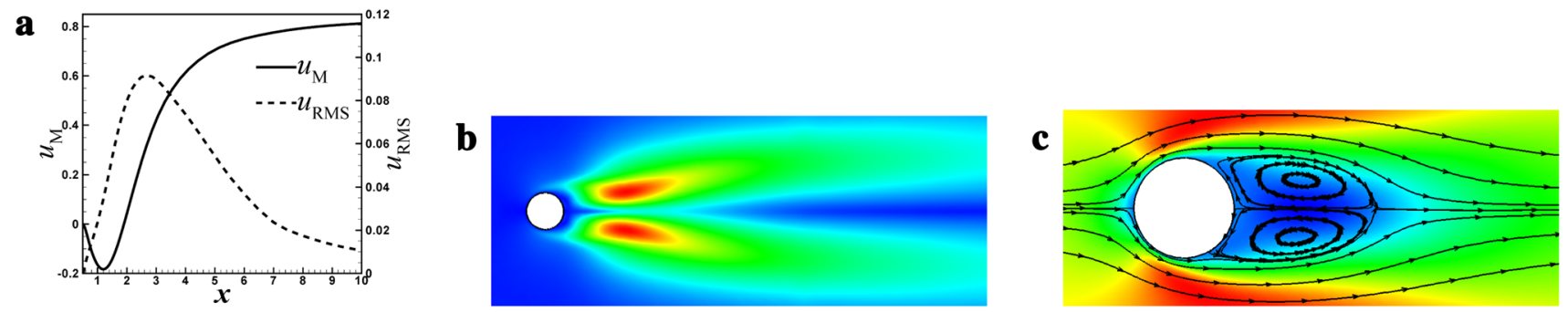

Fig. 4 For the basic case: a non-dimensional RMS streamwise velocity $u_{\text {RMS }}$ and mean streamwise velocity $u_{\mathrm{M}}$ varied along with the downstream distance on the wake center line; $\mathbf{b}$ contours of $u_{\mathrm{RMS}}$ with the maximum of red and minimum of blue; $\mathbf{c}$ streamlines for mean velocity field and contours of $u_{\mathrm{M}}$ near the cylinder with red and blue denoting the positive and negative values, respectively

To illustrate better the vortex-shedding instability and regimes, Fig. 4a presents distributions of non-dimensional RMS and mean streamwise velocities, $u_{\mathrm{RMS}}$ and $u_{\mathrm{M}}$, in the wake center line and near wake. Present calculations give the vortex formation length, $L_{\mathrm{F}}=2.67 \mathrm{D}$, defined by the distance downstream from the cylinder axis to a point where the RMS velocity fluctuations are maximized on the wake center line (see also shown in Fig. 4b), and the bubble length of mean recirculation region in the wake (this region is symmetric and closed, as shown by streamlines in Fig. $4 \mathrm{c}), L_{\mathrm{B}}=1.92 \mathrm{D}$, obtained from the distance between the cylinder center and a point with the zero mean velocity on the wake center line.

\subsection{Streamwise and vertical interactions}

Above all, in order to understand better different vortexshedding patterns due to introduced conic disturbances and peak perforations, it is of importance to introduce two types of interaction between the streamwise or vertical and spanwise vortices. These interactions occur in the boundary layers firstly and then the top and bottom shear layers, where the spanwise vorticity is concentrated and rolled up to form the spanwise vortex beginning to shed, in a simple manner and finally in the near wake with the alternatively shedding spanwise vortices. However, additional components of vorticity on cylinder surfaces, different from those in the shear layers, and the conic disturbance itself would interfere in the analysis. In the near wake, these interactions in different intensities and spacial distributions become very complicated because of viscous diffusion of vorticity, cancelation of vorticity of opposite signs, self-rotation of spanwise vortex, and stretching of vorticity by the oppositely signed vorticity. Thus, it would also be difficult to identify such interactions in the wake, especially for the vertical vs. spanwise vortices. In addition, the interaction of streamwise vs. spanwise vortices could be obtained from the previous results of Meiburg and Lasheras [23]. The main focus is put on the formation of spanwise vortices being about to shed, and accordingly to present the vortex-shedding patterns. Therefore, the interactions of the streamwise vs. spanwise vortices and vertical vs. spanwise vortices are mainly illustrated in the shear layers and the near wake.

At first, let us discuss the interaction between the streamwise and spanwise vortices, referred as to the streamwise interaction. In general, due to specific boundary conditions, only streamwise vortices are generated on a flat plate surface when the normal direction of such plate is aligned with the vertical direction. Then the streamwise interaction could be studied in the wake of the flat plate. Through experiments and numerical simulations, the 3-D structure of plane wake behind the flat plate subjected to periodic spanwise perturbations at a lower Reynolds number $(R e \approx 100)$ has 
a

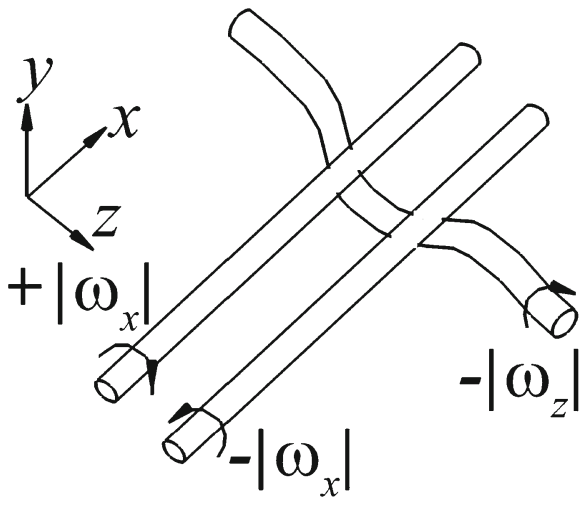

b

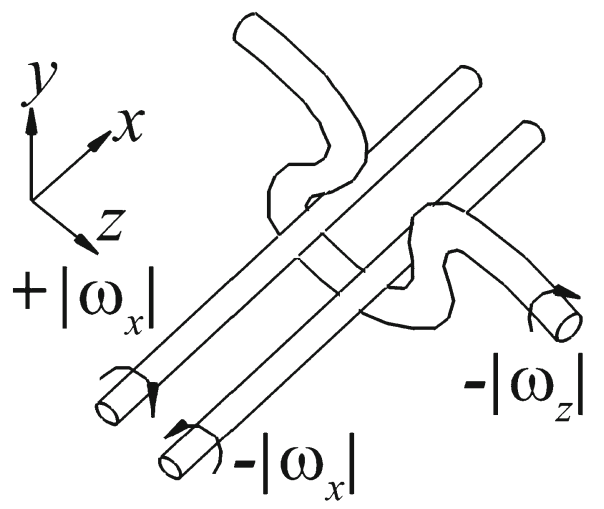

Fig. 5 Sketches of $\mathbf{a}$ weak and $\mathbf{b}$ strong interactions between the streamwise and spanwise vortices
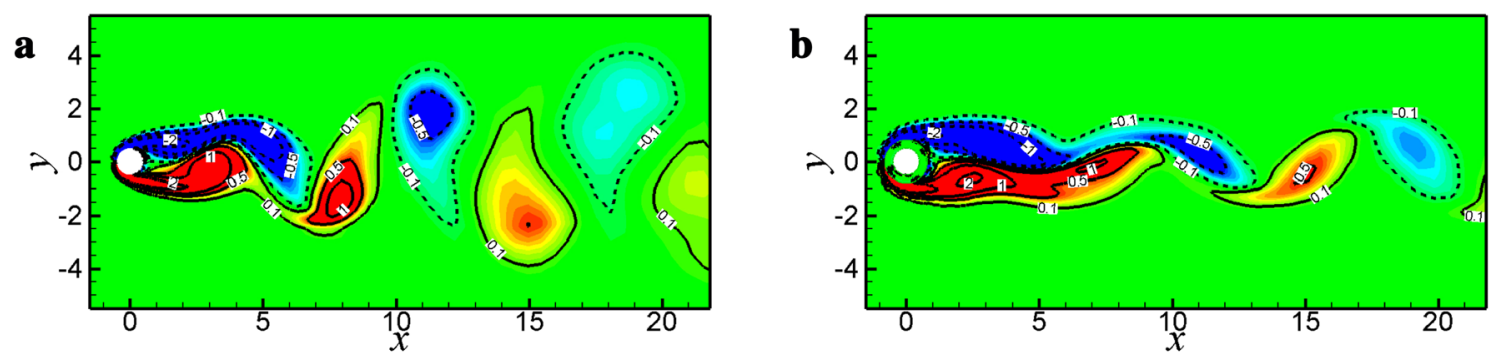

Fig. 6 Contours of spanwise vorticity at $\mathbf{a} z=0$ and $\mathbf{b} \frac{1}{2} \frac{\lambda}{D}$ in the case of peak-perforated conic shroud (marked by "Co") Co: $\lambda / D=6, W / \lambda=$ $0.05, A g=30^{\circ}, D o / D=0.1$. The red and blue, as well as the solid and dashed lines, denote the positive and negative signs of $\omega_{z}$, respectively (same description for the following similar figures)
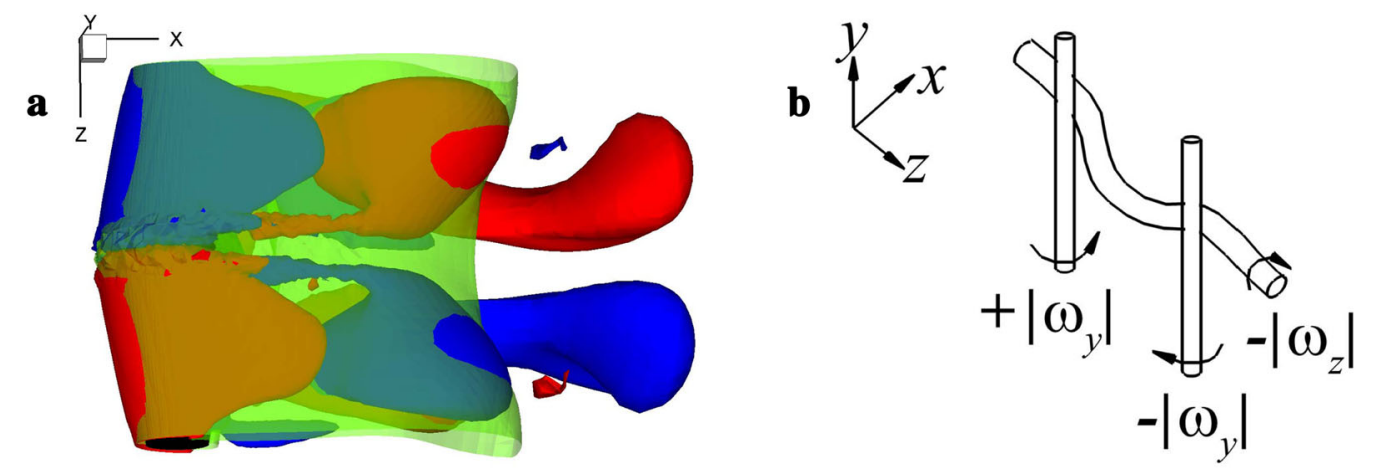

Fig. 7 a Iso-surfaces of vertical and spanwise vorticities in the vortex-formation region in the case of Co: $\lambda / D=6, W / \lambda=0.1, A g=0^{\circ}, D o / D=$ 0.1 at $t=250$, where $\omega_{y}= \pm 0.2$ (red/blue) and $\omega_{z}=-0.5$ (translucent green); b sketch of weak interaction between vertical and spanwise vortices

been already studied by Meiburg and Lasheras [23]. As the streamwise vortex tubes evolve, they interact with the spanwise vortices by lifting them up and pushing them down (in the vertical direction) in an alternating fashion (along the spanwise direction). The resulting curvature of the spanwise vortices, in turn, causes further stretching and intensification of the streamwise vorticity. Thus, the interaction between the streamwise and the spanwise vortices proceeds in a selfamplifying manner, as shown in Fig. 5 b.

Based on this analysis and in consideration of the present results, the weak and strong streamwise interactions are iden- tified, as shown in Fig. 5, when the streamwise vortex pairs become weak and strong relatively compared to the spanwise vortices, respectively. Among them, the main feature in the strong streamwise interaction is the violently curved extent and consequently greatly amplified cross-sectional area (normal to the spanwise direction) of spanwise vortices. As shown in Fig. 6 when the spanwise vortices are described by the iso-surface of spanwise vorticity, the spanwise vorticity is diffused in a large region at $z=0$ (for example, $\omega_{z}= \pm 0.1$ in range of $y= \pm 4$ ) but gathered in a small region near the wake center plane at $z=\frac{1}{2} \frac{\lambda}{D}$ 
(within \pm 2 ), compared with that in the basic case in Fig. 3a (within \pm 2.5 ). Besides, the strong streamwise interaction of the evolving streamwise structure with the spanwise Kármán vortices results in the formation of closed vortex loops of alternating sign [23].

Correspondingly, the interaction between the vertical and spanwise vortices, referred to as the vertical interaction, results in the forward and backward distortion of the spanwise vortices (in the streamwise direction) alternatively (along the spanwise direction). There are also weak and strong vertical interactions. Numerical simulations present such vertical interaction in the upper shear layer with the formation of spanwise vortex, as shown in Fig. 7a and sketched in Fig. 7b. Particularly in certain circumstances, sub-regime III-C described in Sect. 3.3, this vertical interaction with the weak streamwise vorticity causes the stretching of shear layers downstream to the far wake and results in the total suppression of Kármán vortex shedding [16,20]. The strong streamwise vorticity is similar to the strong streamwise interaction in Fig. 5b. Hereby, the violent curvature of shear layer along the span leads to the formation of spatially-curved spanwise vortex.

In a certain case, sub-regime III-A (type A) presented in Sect. 3.3, when the vortex-shedding frequencies at different spanwise positions are still same, the spatially curved effect in the $(x, z)$ plane due to strong vertical interaction is effectively equivalent to the phase difference in time for the dragging effect of vertical vortex pairs on spanwise vortex. Given that the periodically shedding spanwise vorticity in the 2-D flow field is $\omega_{z}(t ; x, y)=A(x, y) \mathrm{e}^{\mathrm{i} 2 \pi f t}$ with already known 2-D spatial distribution function $A(x, y)$, the introduction of conic disturbance and resultant generation of vertical vorticity result in the 2-D spanwise vorticity distorted uniformly (assumed to be) along the span with the wavelength $\lambda$. Then, we have $\omega_{z}(t ; x, y, z)=A(x, y) \mathrm{e}^{\mathrm{i} 2 \pi f t} \mathrm{e}^{\mathrm{i} \frac{2 \pi}{\lambda / D} z}$. It can be obtained that $\omega_{z}=A(x, y) \mathrm{e}^{\mathrm{i} 2 \pi f t}$ at $z=0$, but at $z=\frac{1}{2} \frac{\lambda}{D}, \omega_{z}=A(x, y) \mathrm{e}^{\mathrm{i} 2 \pi f t} \mathrm{e}^{\mathrm{i} \pi}=A(x, y) \mathrm{e}^{\mathrm{i}(2 \pi f t+\pi)}$. This shows that the spanwise vortex at $z=\frac{1}{2} \frac{\lambda}{D}$ seems to be dragged until half a period later and then shed into the wake. The spanwise vortices at $z=0$ and $\frac{1}{2} \frac{\lambda}{D}$ are just $180^{\circ}$ out-of-phase in time or spanwise space at the same time.

\subsection{Reexamination of vortex-shedding patterns in the near wake}

As reported in previous works [16,21], there are three different flow regimes based on different intensity of disturbances at $R e=100$. Regimes I, II, and III are related to the weak, moderate and strong disturbances, i.e., conic disturbances with and without peak perforations. Let us reexamine different vortex-shedding patterns through the analysis from the view point of above streamwise and vertical interactions.
(Regime I) In regime I with the weak disturbance, the near wake can still be described by the alternatively shedding Kármán or Kármán-like vortex street occurred in the wake of 2-D circular cylinder, as shown in Figs. 8 and 9. The generated streamwise and vertical vortices themselves are very weak and quickly dissipated within the vortex-formation region (in sub-regime I-A), or shed with the shedding spanwise vortices within a certain downstream distance (in sub-regime I-B). As a result, the streamwise and/or vertical interactions in the upper and lower shear layers are so weak that both layers and shedding spanwise vortices are little wavily distorted across the span.

(Regime II) As for the moderate effect of disturbance in regime II, typically as shown in Figs. 10 and 11 in sub-regime II-A, and Figs. 12 and 13 in sub-regime II-B, the shedding spanwise vortices, as well as shear layers, are wavily distorted and non-uniformly in the vertical-spanwise plane. In spite of this, they are still shed alternatively downstream and almost kept synchronization along the spanwise direction. In subregime II-A, spanwise vortices are distorted mainly by the streamwise vortex pairs or due to the streamwise interaction. In sub-regime II-B, spanwise vortices are distorted by both streamwise and vertical vortex pairs or under coupled effects of streamwise and vertical interactions. The shear layer in present sub-regime stretches downstream further than that in sub-regime II-A. For instance, at $z=\frac{1}{4} \frac{\lambda}{D}$, contours of $\omega_{z}=-0.5$ extend downstream about 6 in Fig. 14 but 7.8 in Fig. 15. In addition, in the present calculational parameters, this vortex-shedding pattern, the distorted spanwise vortex coupled with one pair of streamwise and vertical vortices with opposite signs alternatively shed in the wake, is very similar to the П-type vortex [20] of the mode A in the 3-D wake transition of the circular or square-section cylinder at moderate Reynolds numbers (see in Ref. [19]), as shown in Fig. 16. As for the П-type vortex itself, the head line "_." denotes the spanwise vortex, while two legs "| |" denote streamwise and/or vertical vortex pairs shed with this spanwise vortex and elongated by the upstream spanwise vortex of opposite sign.

(Regime III) As the effect of disturbance becomes strong in regime III, the main feature is that the original Kármán or Kármán-like vortex shedding is totally disappeared or just appeared occasionally in coexistence with other different vortex-shedding patterns. Such phenomenon could be related to additional components of vorticity with complex spacial distributions and different strengthes, prominently different from those with regular signs under the moderate effect, and subsequently coupled streamwise and vertical interactions between the streamwise and vertical vortices and spanwise vortices.

In sub-regime III-A, the wake flow is mainly described by the appearance of the $\Omega$-type vortex shedding at the valley or peak, typically as shown in Figs. 17 and 18 in type A, 
$\mathbf{a}$

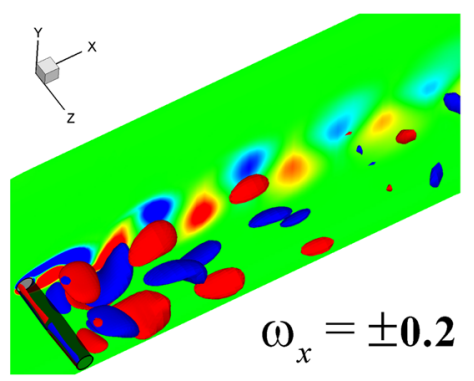

b

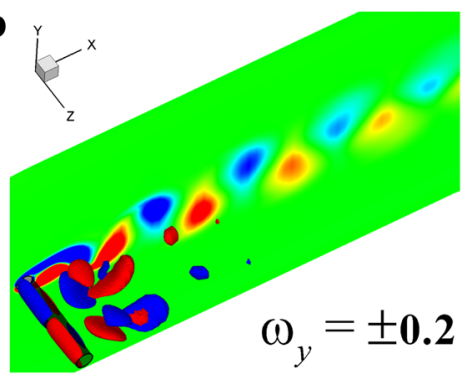

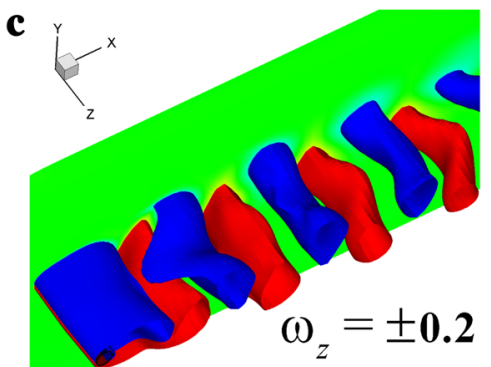

Fig. 8 Iso-surfaces of three components of vorticity, $\omega_{x}, \omega_{y}$, and $\omega_{z}$, in the near wake at $t=265$ in the case of the conic shroud (marked by "C") (C: $\lambda / D=6, W / \lambda=0.0125$ ), in the background of which is sectional contours of $\omega_{z}$ at $z=0$, where red and blue in the contours and iso-surfaces indicate positive and negative values (same description for the following similar figures). Note that the body is shown by using semi-transparent black surface and a wire frame

$\mathbf{a}$

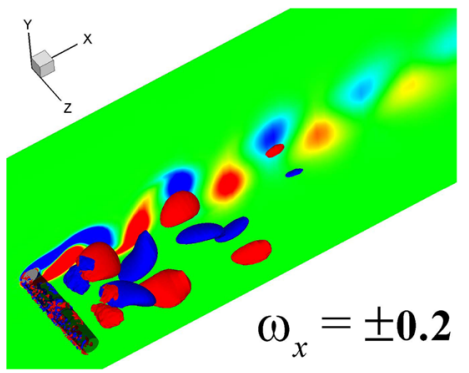

b

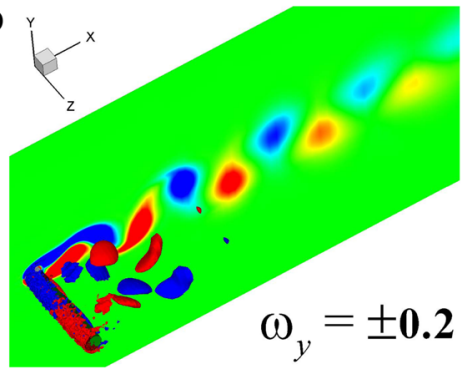

c

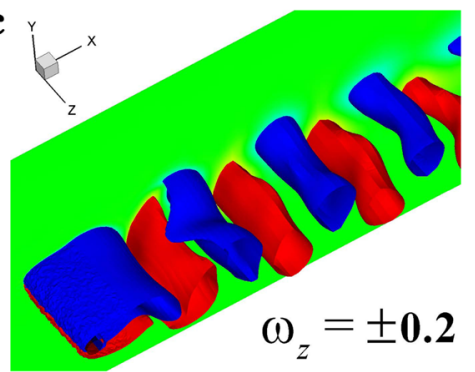

Fig. 9 Iso-surfaces of three components of vorticity, $\omega_{x}, \omega_{y}$, and $\omega_{z}$, in the near wake in the case of Co: $\lambda / D=6, W / \lambda=0.0125, A g=$ $0^{\circ}, D o / D=0.4$. Note that the body is shown by using semi-transparent black surface and a wire frame

$\mathbf{a}$

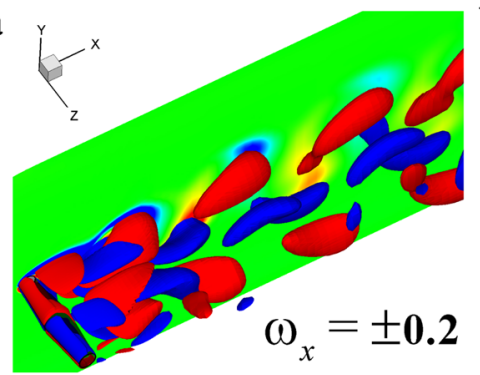

b

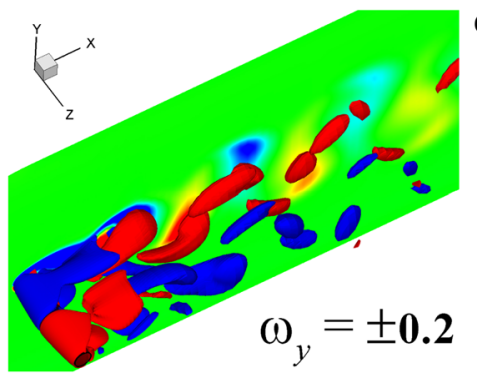

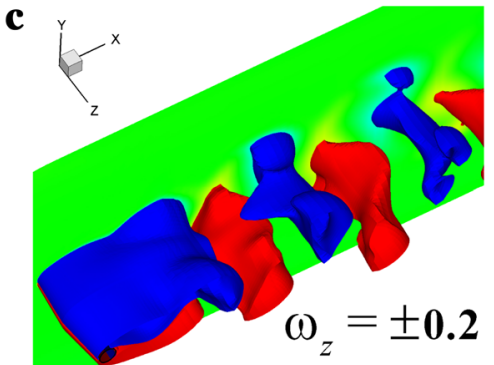

Fig. 10 Iso-surfaces of $\omega_{x}, \omega_{y}$, and $\omega_{z}$ in the near wake at $t=1950$ in the case of C: $\lambda / D=6, W / \lambda=0.05$

a

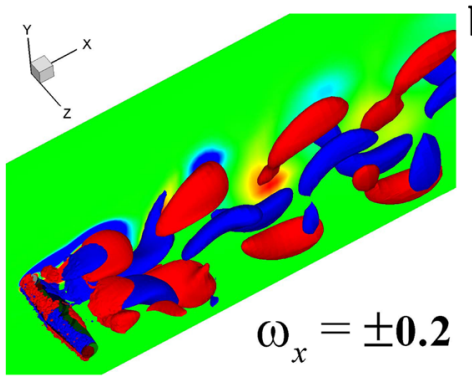

b

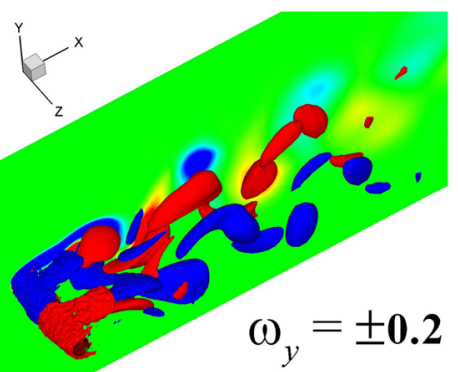

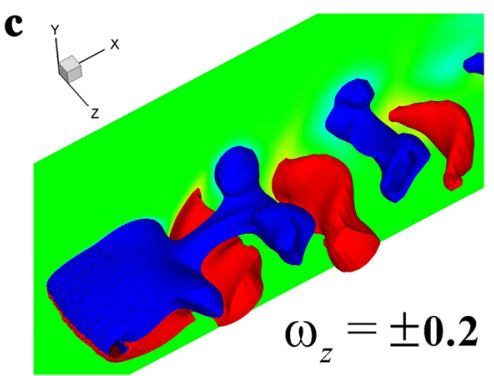

Fig. 11 Iso-surfaces of $\omega_{x}, \omega_{y}$, and $\omega_{z}$ in the near wake at $t=780$ in the case of Co: $\lambda / D=6, W / \lambda=0.05, A g=30^{\circ}, D o / D=0.1$ 
$\mathbf{a}$

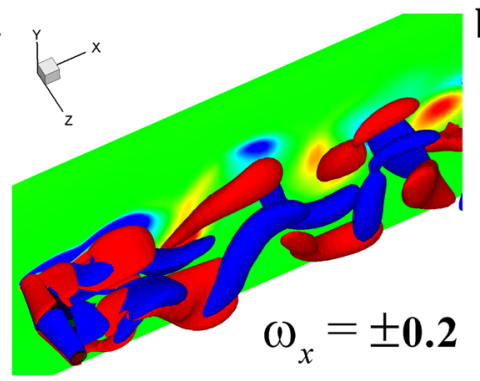

b

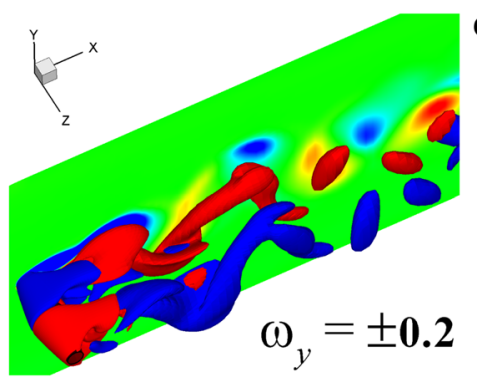

c

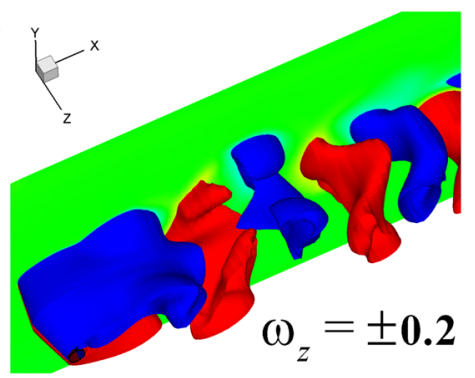

Fig. 12 Iso-surfaces of $\omega_{x}, \omega_{y}$, and $\omega_{z}$ in the near wake at $t=380$ in the case of $\mathrm{C}: \lambda / D=6, W / \lambda=0.1$

$\mathbf{a}$

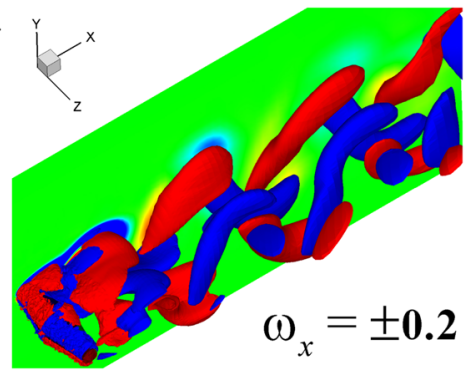

b

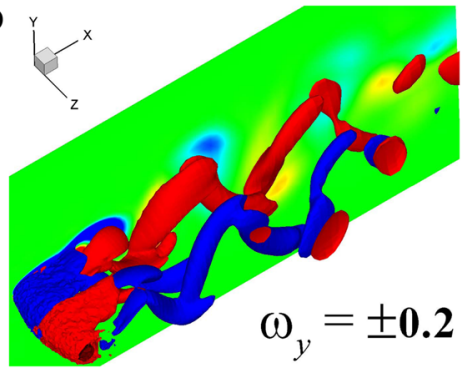

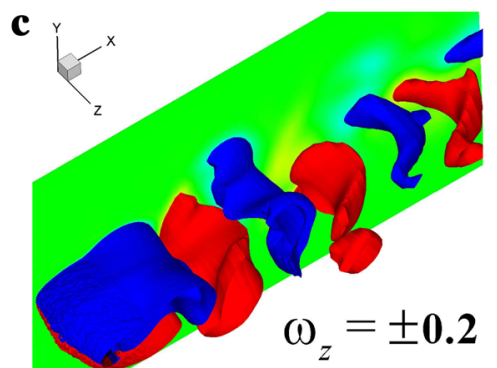

Fig. 13 Iso-surfaces of $\omega_{x}, \omega_{y}$, and $\omega_{z}$ in the near wake at $t=600$ in the case of Co: $\lambda / D=6, W / \lambda=0.1, A g=0^{\circ}, D o / D=0.4$
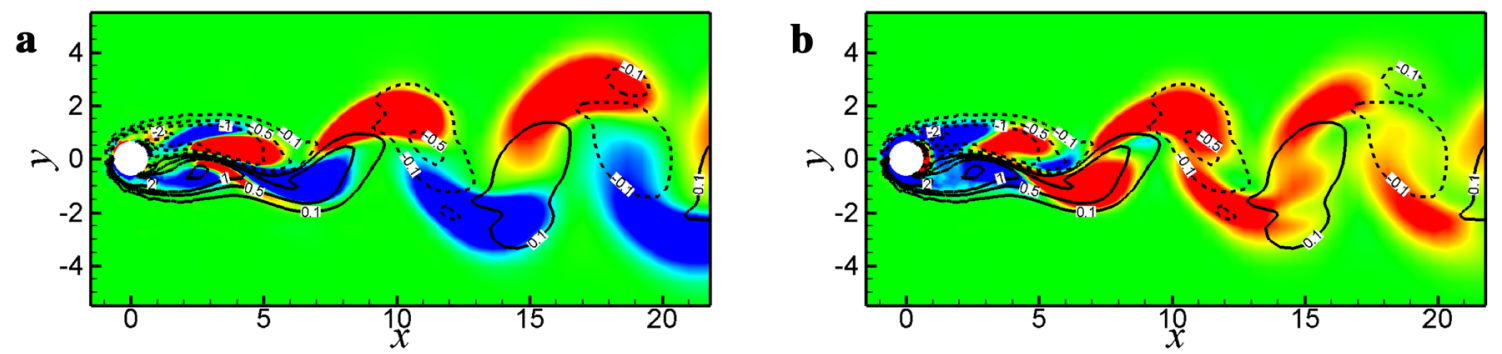

Fig. 14 Contours of $\mathbf{a} \omega_{x}$ and $\mathbf{b} \omega_{y}$ at $t=780$ and $z=\frac{1}{4} \frac{\lambda}{D}$ in the case of Fig. 11, where red and blue denote the positive and negative values, respectively, and the solid and dashed lines denote the contours of spanwise vorticity
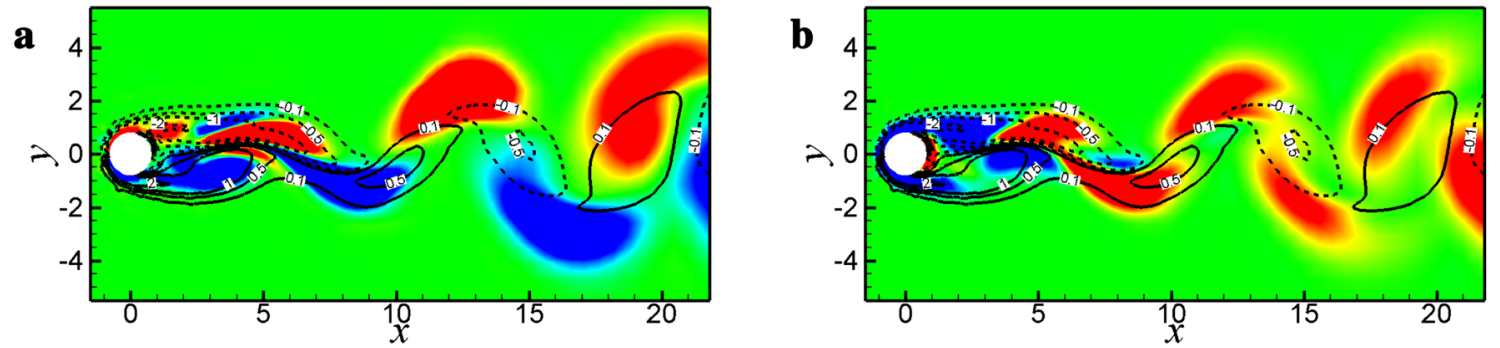

Fig. 15 Contours of $\mathbf{a} \omega_{x}$ and $\mathbf{b} \omega_{y}$ at $t=600$ and $z=\frac{1}{4} \frac{\lambda}{D}$ in the case of Fig. 13, where red and blue denote the positive and negative values, respectively, and the solid and dashed lines denote the contours of spanwise vorticity

Fig. 19 in type B, and Fig. 20 in type C. It can be seen that the noteworthy feature is that at a certain spanwise position (such as $z=\frac{1}{4} \frac{\lambda}{D}$ ), the oppositely signed streamwise vorticity is alternatively distributed in the upper or lower shear layer, as well as the oppositely signed vertical vorticity downstream away from the vortex-formation region. For example, from the point of view based on the vertical interaction in Fig. 7, such specific distribution of $\omega_{y}$ determines a row of spanwise vortices with same sign at different spanwise positions shed in a $180^{\circ}$ out-of-phase, as sketched in Fig. 21. 

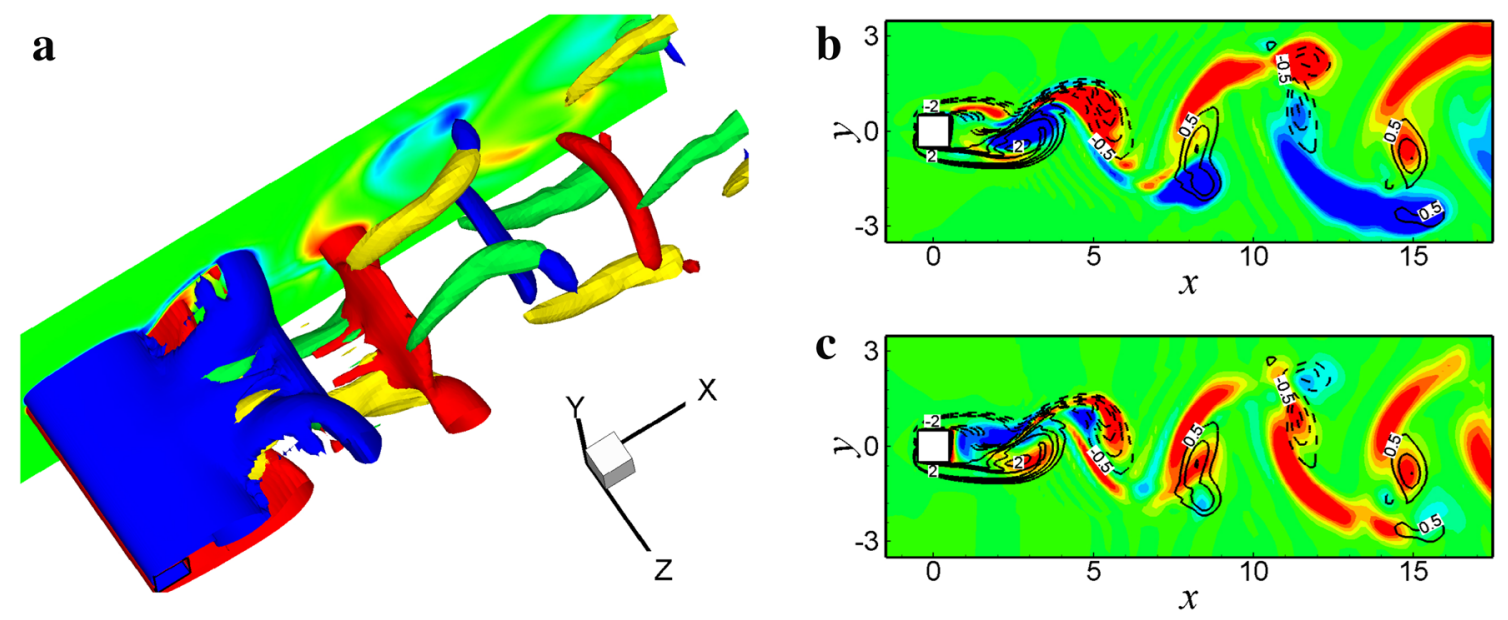

Fig. 16 a Iso-surfaces of $\omega_{x}= \pm 0.8$ (yellow/green) and $\omega_{z}= \pm 1$ (red/blue) in the near wake of the square-section cylinder at $t=340$ and $R e=180$ with non-dimensional spanwise computational length of 6 ; contours of $\mathbf{b} \omega_{x}$ and $\mathbf{c} \omega_{y}$ at $z=1.5$ where red and blue denote the positive and negative values, as well as the solid and dashed lines in contours of spanwise vorticity, respectively. Note that the body is shown by a wire frame
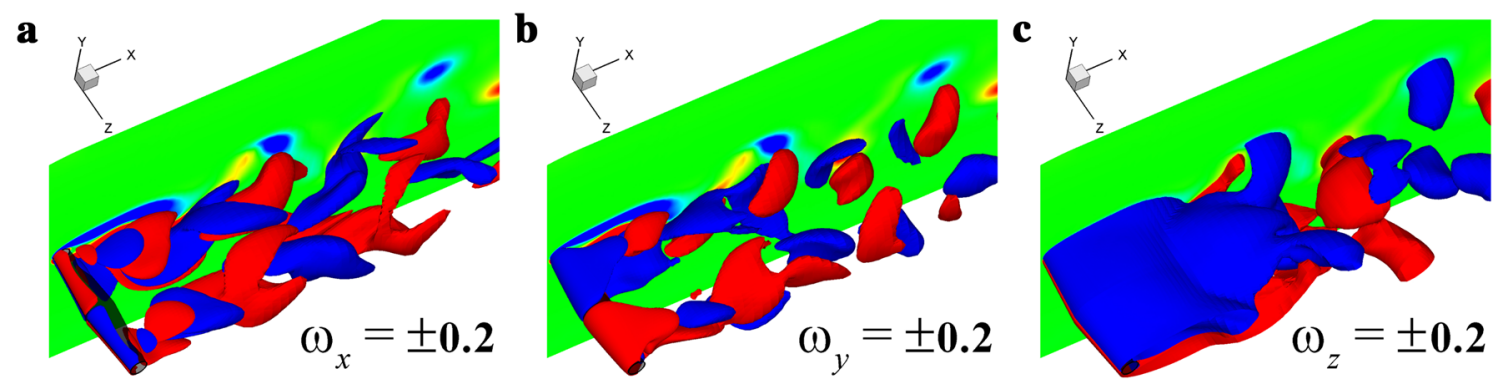

Fig. 17 Iso-surfaces of $\omega_{x}, \omega_{y}$, and $\omega_{z}$ in the near wake at $t=880$ in the case of C: $\lambda / D=8, W / \lambda=0.05$
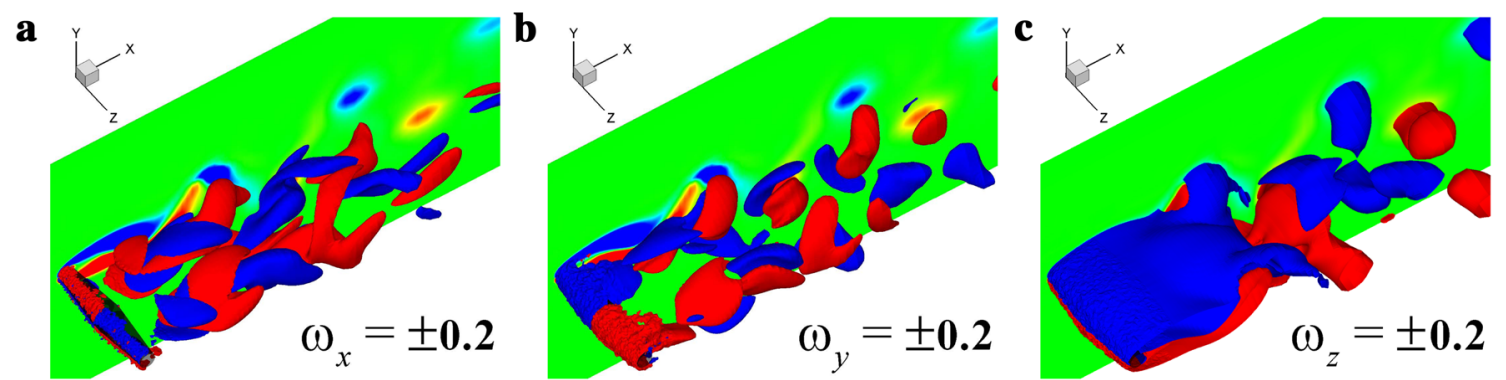

Fig. 18 Iso-surfaces of $\omega_{x}, \omega_{y}$, and $\omega_{z}$ in the near wake at $t=900$ in the case of Co: $\lambda / D=8, W / \lambda=0.05, A g=30^{\circ}, D o / D=0.1$

In sub-regime III-A (types A and B), Fig. 21a presents the forming process of $\Omega$-type vortex shed at the valley. The first vertical vortex pair within the vortex-formation region, which obviously do not originate from the front surface of the cylinder because of $\omega_{y}$ with opposite signs in Figs. 17 and 18, are strongly interacted with the shear layers being about to be concentrated and rolled up into the shedding spanwise vortices. Therefore, it effectively "drags" the spanwise vortex at the peak until the spanwise vortex at the valley is totally shed into the wake. In addition, due to the existence of the second vertical vortex pair (with signs just contrary to the first pair), which actually originates from the front surface because of same signs, the opposite effect of these vertical vortices leads to the downstream already-shed spanwise vortex at the peak further "pushed" downstream and the newly formed spanwise vortex at the valley "suppressed" until the effect of the first vertical vortex pair also newly formed in the shear layer becomes strong enough and then "pushes" the spanwise vor- 
a

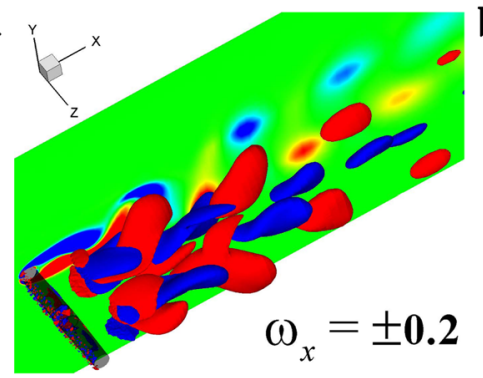

b

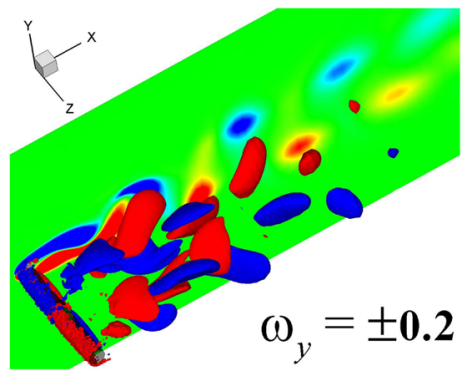

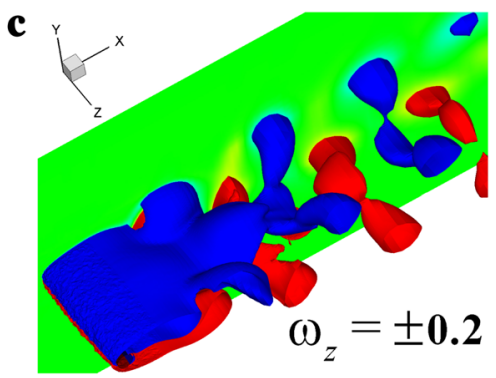

Fig. 19 Iso-surfaces of $\omega_{x}, \omega_{y}$, and $\omega_{z}$ in the near wake at $t=470$ in the case of Co: $\lambda / D=8, W / \lambda=0.0125, A g=0^{\circ}, D o / D=0.4$

a

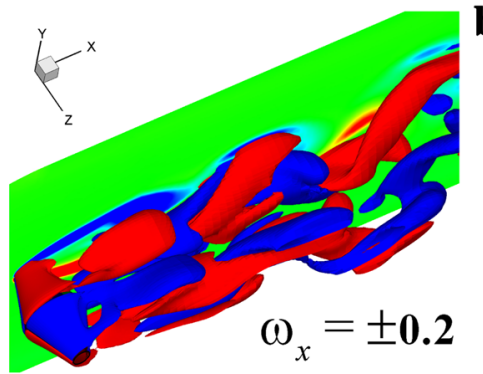

b

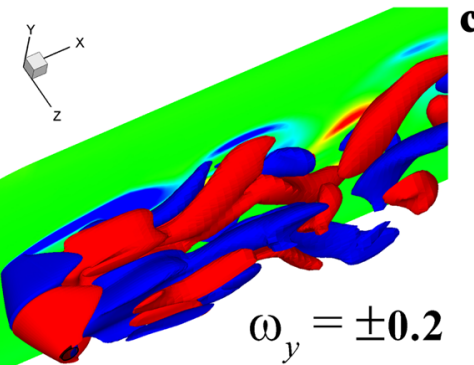

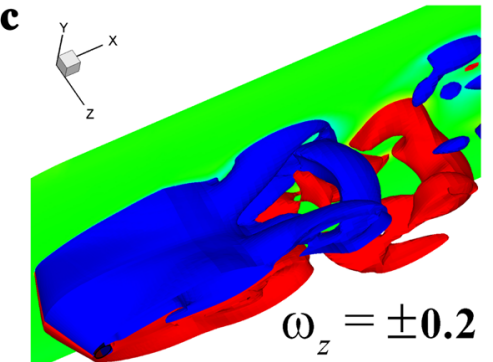

Fig. 20 Iso-surfaces of $\omega_{x}, \omega_{y}$, and $\omega_{z}$ in the near wake at $t=1200$ in the case of $\mathrm{C}: \lambda / D=6, W / \lambda=0.2$

a

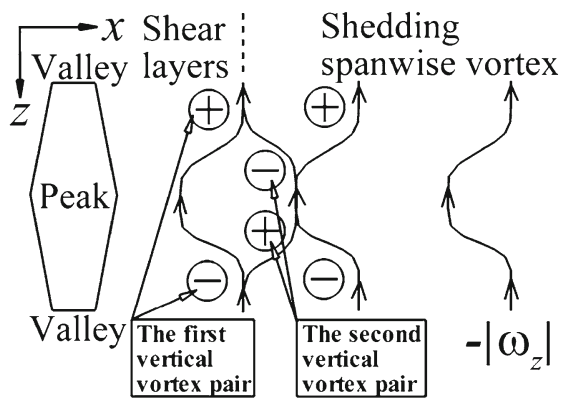

b

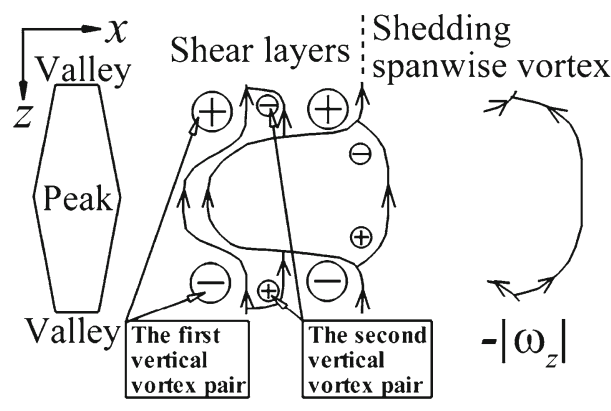

Fig. 21 Sketches of $\Omega$-type vortices shed at the $\mathbf{a}$ valley and $\mathbf{b}$ peak illustrating the strong vertical interaction between the vertical and negative spanwise vortices, where + and - denote the positive and negative signs of $\omega_{y}$ respectively, and $\circ$ and $\bigcirc$ denote the small and large spacial regions obtained from the $\omega_{y}$ iso-surfaces, respectively. The dashed line is just used to differentiate region of shear layers from that of shedding vortex in the wake approximately

a

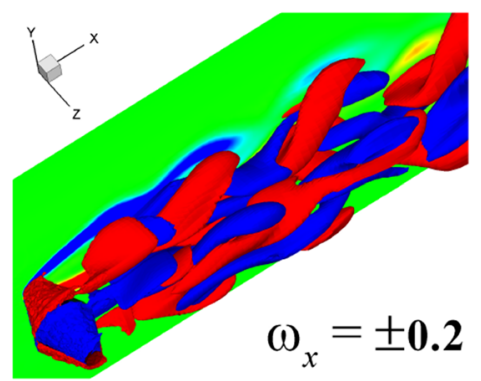

b

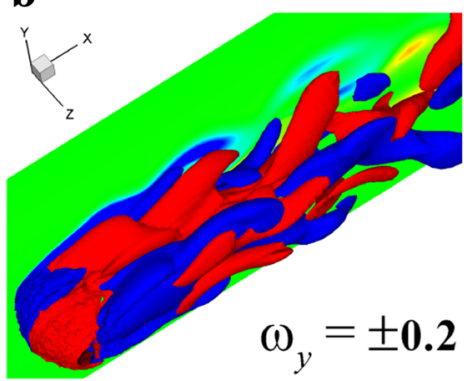

c

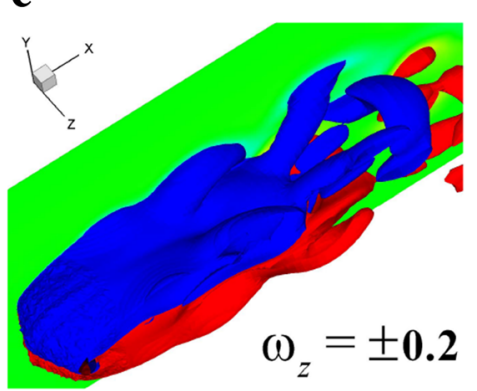

Fig. 22 Iso-surfaces of $\omega_{x}, \omega_{y}$, and $\omega_{z}$ in the near wake at $t=200$ in the case of Co: $\lambda / D=6, W / \lambda=0.2, A g=0^{\circ}, D o / D=0.1$ 
a

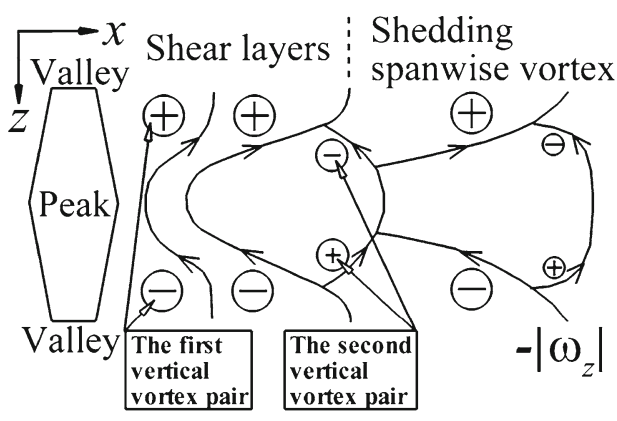

b

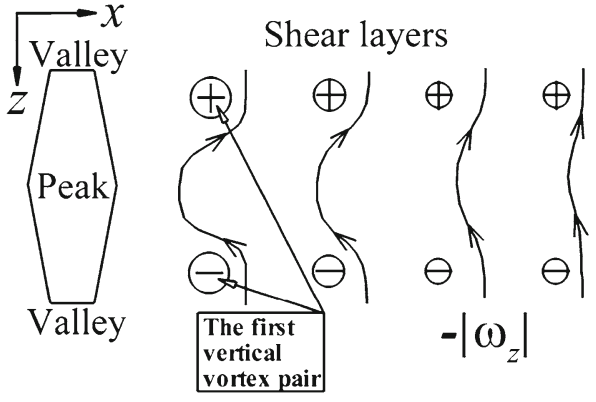

Fig. 23 Sketches of a the cloud-like vortex: $\Omega$-type vortices alternatively shed at the peak and valley and $\mathbf{b}$ the complete suppression of Kármán vortex shedding

tex downstream to be shed at the valley in a new period. For the primary effect of the first vertical vortices, the shedding spanwise vortex mainly looks like the $\Omega$ form curved in the center of the valley and heading to downstream, called as the $\Omega$-type vortex shed at the valley, similar to the hairpin vortex.

However, in sub-regime III-A (type C), the $\Omega$-type vortex is mainly shed at the peak. The formation of the $\Omega$-type vortex shed at the peak undertakes a very similar process, as shown in Fig. 21b. By comparing to the $\Omega$-type vortex shed at the valley, the obvious feature is that the first vertical vortex pair is greater or stronger than the second in both spatial distributions and intensities. As a result, the strong vertical interaction of the first vertical vortices in the shear layers gives rise to the shear layers violently distorted upstream. Therefore, the spanwise vortices are hardly shed at the peak and the shear layers are elongated greatly far away downstream at the valley. Until the second vertical vortex pair appears and separates the part of spanwise vorticity in the shear layer near the valley, the downstream spanwise vorticity at the valley is thus concentrated and the spanwise vortex is formed to be shed with the already-shed spanwise vortex downstream at the peak. After this process, the upstream spanwise vortices at the peak are then pushed to be newly formed and shed under the effect of the second vertical vortex pair, but still connected with the newly grown shear layers at the valley. Taken into account of the already-shed spanwise vortices downstream at the peak, the $\Omega$-type vortex shed at the peak is described by the formation with its top orientated to the center of the peak. The main difference, compared to the pattern in type A, just lies in that the shedding spanwise vortices at the valley are distorted so violently that they are only distributed near the valley, and shed along with the already-shed spanwise vortices at the peak.

As for sub-regime III-B, a cloud-like vortex, which actually is the $\Omega$-type vortex alternatively shed at the peak and valley, as shown in Fig. 22, mainly appears in the near wake at present disturbed parameters. The shear layers are wavily varied along both the streamwise and spanwise directions for the sake of complex distributions of streamwise and vertical vortices. Furthermore in the vortex-shedding region, these shedding spanwise vortices under coupled effects of the first and second vertical vortex pairs are actually bent toward downstream at the peak and valley in an alternative way during the whole vortex-shedding procession, as sketched in Fig. 23a.

In sub-regime III-C, shear layers are elongated far away downstream and Kármán vortex shedding is completely suppressed, as shown in Figs. 24 and 25, and its formation process is sketched in Fig. 23b. The strong conic disturbance, even with peak perforation, leads to the specific spanwise flow near the body, especially the rear surface. As a result, the vertical vorticity is generated on the wall and induced in the shear layers with opposite signs. Apparently different from previous cases, the second vertical vortex pair is totally suppressed in the wake and only the first vertical vortex pair is dominant and gradually expanded in the shear layers. Therefore, the original spanwise vortices are distorted along the same direction or almost parallel to each other. On the other hand, the fact that the vertical vorticity is obviously much larger than the streamwise vorticity in the whole wake flow indicates that the vertical interaction is also dominant in the present cases. Meanwhile the strong vertical interaction results in the wake flow mainly turning around in the $(x, z)$ plane with the formation of two recirculating regions as shown in Fig. 26, rather than in the $(x, y)$ plane traditionally with the Kármán vortex shedding. Because of the separation effect of recirculation, top and bottom shear layers cannot interact with each other and subsequently stretch far away downstream. The wake flow becomes steady if without peak perforation. Moreover, also as shown in Fig. 26b, a tiny jet (shown by distribution of vertical vorticity near the hole) introduced from the perforation on the rear surface just perturbs the symmetry of two recirculation zones with the peak, thus having little effect on above suppression mechanism and flow characteristics.

Except for these four basic cases, in sub-regime III-D, multiple vortex-shedding patterns alternatively appearing or coexisting with the $\Omega$-type vortex indicate complex coupled effects of streamwise and vertical interactions in the near wake. They are mainly observed in the flow of 
$\mathbf{a}$

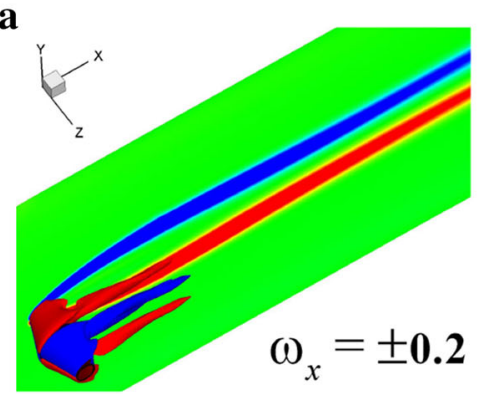

b

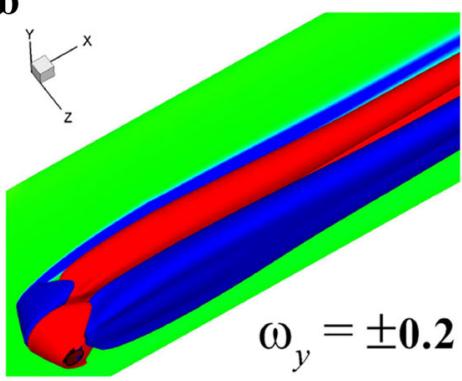

c

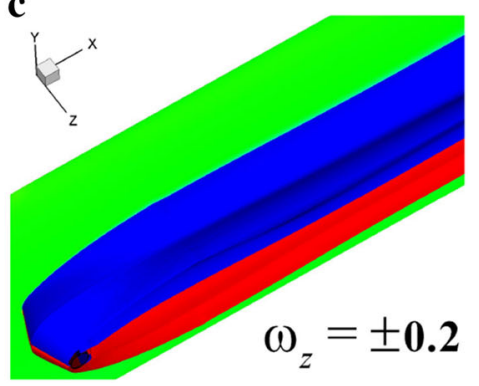

Fig. 24 Iso-surfaces of $\omega_{x}, \omega_{y}$, and $\omega_{z}$ in the near wake at $t=230$ in the case of $\mathrm{C}: \lambda / D=4, W / \lambda=0.2$

a

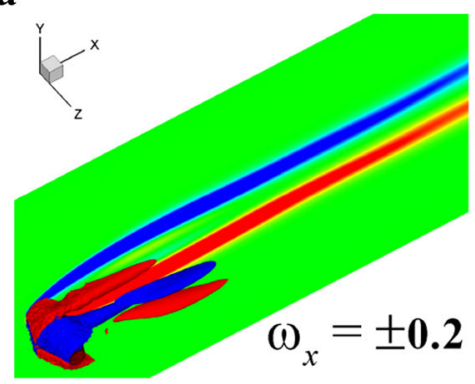

b

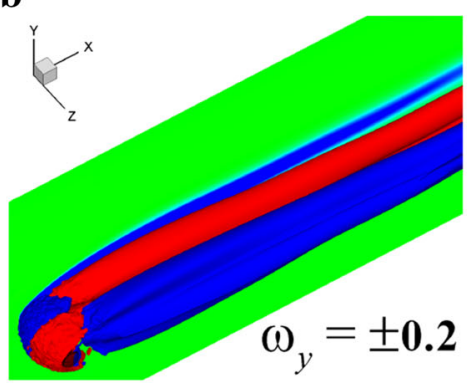

c

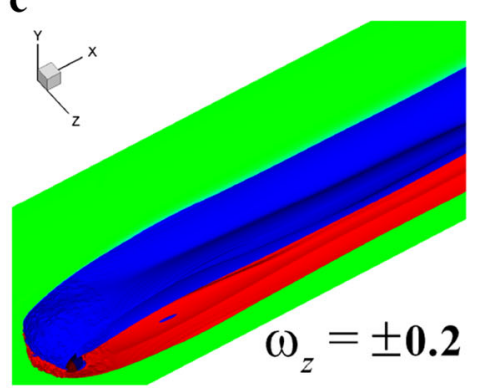

Fig. 25 Iso-surfaces of $\omega_{x}, \omega_{y}$, and $\omega_{z}$ in the near wake at $t=700$ in the case of Co: $\lambda / D=4, W / \lambda=0.2, A g=0^{\circ}, D o / D=0.2$

a

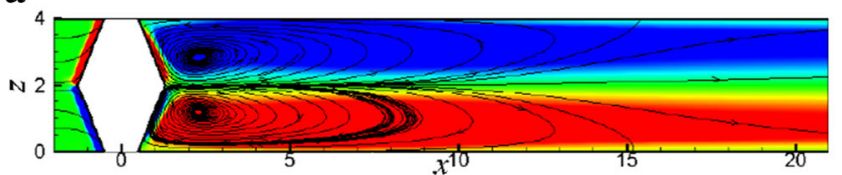

b

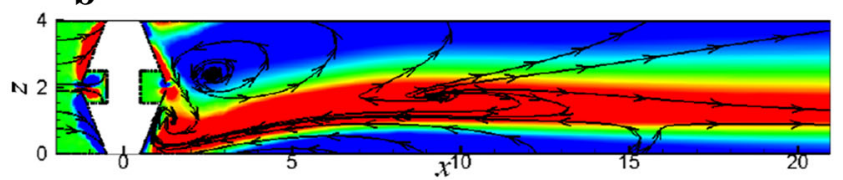

Fig. 26 Contours of $\omega_{y}$ and streamlines in the wake center plane $(y=0)$ at $\mathbf{a} t=230$ and $\mathbf{b} t=700$ in cases of Figs. 24 and 25 , respectively, where the red and blue denote the positive and negative values of vertical vorticity respectively

a

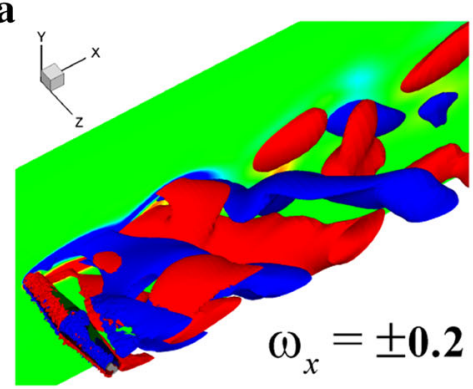

b

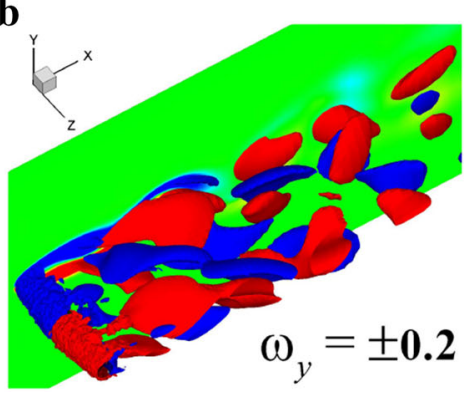

c

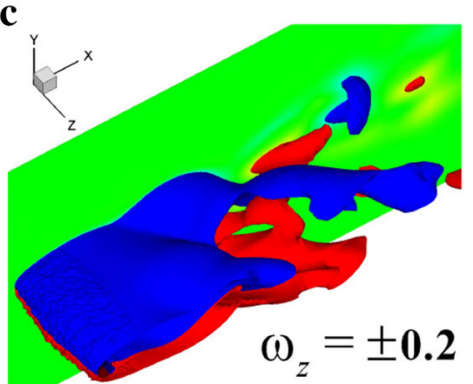

Fig. 27 Iso-surfaces of $\omega_{x}, \omega_{y}$, and $\omega_{z}$ in the near wake at $t=1580$ in the case of Co: $\lambda / D=8, W / \lambda=0.05, A g=0^{\circ}, D o / D=0.1$

peak-perforated conic shrouds. So far, for the present computational parameter space, except for the typical $\Omega$-type vortex-shedding pattern stated previously appearing in the near wake, two other complicated vortex-shedding patterns are identified. One is the obliquely shedding vortex at the lowest fluid forces, appearing as a competitive pattern in type A, as shown in Fig. 27. The typical oblique angle to the axis of the cylinder is about $\pm 38^{\circ}$. Another is the crossed spanwise vortex with opposite signs only existing as a transitional pattern in a series of successive vortex-shedding patterns in type B, as shown in Fig. 28. The spanwise vortices with a positive sign extend slantly along the cylinder axis and upwards into the upper side of wake $y>0$, like a thorn. Meanwhile the upper shear layer at the peak stretches downwards into the lower side of wake $y<0$ due to the strong streamwise interaction, and the spanwise vortices with a negative sign 
a

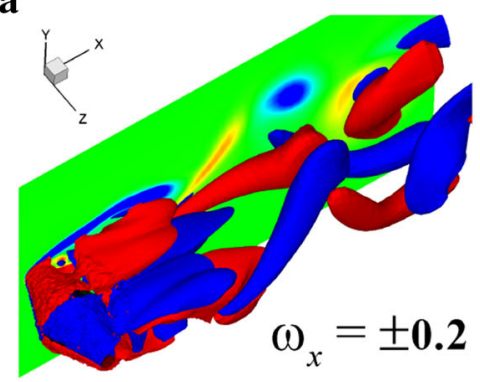

b

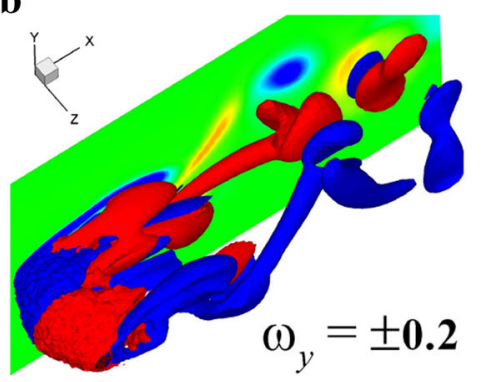

c

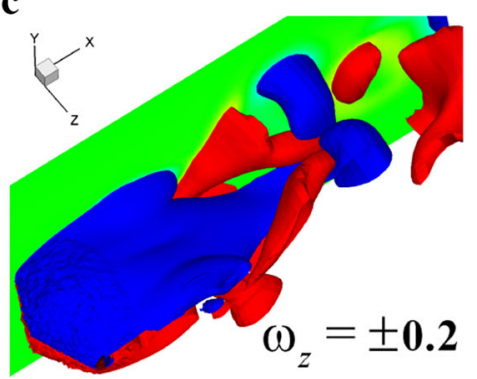

Fig. 28 Iso-surfaces of $\omega_{x}, \omega_{y}$, and $\omega_{z}$ in the near wake at $t=420$ in the case of Co: $\lambda / D=8, W / \lambda=0.2, A g=0^{\circ}, D o / D=0.1$

near the valley are still regularly shed into the wake. From the point of spacial distributions of three components of vorticity, the obliquely shedding vortex is mainly influenced by the streamwise vortices or streamwise interaction, while the crossed spanwise vortices are subjected to both streamwise and vertical vortices or coupled strong streamwise and vertical interactions.

In a summary, based on the analysis for different vortexshedding patterns, there are two new points of view. At present, several sub-regimes (maybe more) of the strong effect of disturbance, the difference of vortex-shedding patterns can be attributed to the competition between the first and second vertical vortex pairs in the shear layers and near wake. For example, if the first vertical vortex pair is equivalent to the second one in spacial range and intensity, the $\Omega$-type vortex shed at the valley appears. While the first vertical vortex pair is dominated, the near wake is mainly described by the $\Omega$ type vortex shed at the peak or alternatively shed at the peak and valley. In the extreme condition that the second vertical vortex pair is eliminated or suppressed due to the suppression mechanism, the strong effect of the first vertical vortex pair leads to the original vortex shedding totally suppressed.

Another view is the competition between the streamwise and vertical interactions with the spanwise vortices used to identify the present several regimes (also maybe more) for different effects of disturbance. For instance, in regime I of weak disturbance, there are both weak streamwise and vertical interactions. But when the streamwise interactions become important, the wake develops into the sub-regime IIA. In the transition from the sub-regime II-B to the regime III (A to C), the vertical interactions gradually play an important role in the evolution of vortex dynamics in the near wake.

\subsection{Wavy shear layers with near-wake width variation across the span}

In the above analysis of vortex-shedding patterns in the flow past the (peak-perforated) conic shroud, it is worth noting that there exists an interesting phenomenon in regime II, which is simply the spacial distribution of streamwise and vertical components of vorticity in the near wake. This phenomenon also appears in the $\Pi$-type vortex of mode $\mathrm{A}$ in the flow past the straight square cylinder at $R e=180$, as shown in Fig. 16. However, as we know up to now, there is no reasonable explanation for this phenomenon. In the following analysis, it will be illustrated and discussed in detail from the following two aspects, wavy shear layers and spacial distributions of three components of vorticity, then sign laws for three components of vorticity in the near wake are obtained.

Above all, let us review two flow characteristics in regime II with the moderate disturbance, including the shear layers and near wake.

The first is the further enhanced streamwise and vertical components of vorticity with specific spatial distributions and their signs, not only on cylinder surfaces but also in the wake. At a certain spanwise position, such as $z=\frac{1}{4} \frac{\lambda}{D}$ or $\frac{3}{4} \frac{\lambda}{D}$, the streamwise vortex pairs with opposite signs are shed alternatively from the upper and lower shear layers throughout the whole computational domain $(30 D)$, or the streamwise vortex with a specific sign is predominantly shed from the upper or lower shear layer, but the vertical vortices substantially with a singular sign are shed from both the upper and lower shear layers. For example, as shown in Figs. 14 and 15 at $z=\frac{1}{4} \frac{\lambda}{D}$, the positive $\omega_{x}$ in the upper shear layer and the negative one in the lower shear layer are concentrated and shed with the negative and positive $\omega_{z}$, respectively; while primarily positive $\omega_{y}$ in both the upper and lower shear layers are appeared and shed. This feature is also found in the near wake of the straight square-section cylinder at $R e=180$ for the successively shedding П-type vortices in mode A [20], as shown in Fig. 16. Because only one wavelength of conic disturbance along the spanwise direction in computations, the additional components of vorticity are basically varied once in opposite signs or with one spanwise wavelength along the span.

The second is the elongated shear layers stretching downstream and obviously distorting wavily along the span. For example, if the shear layer is approximately illustrated by contours of $\omega_{z}$, then contours of $\omega_{z}$ at -0.5 and -1 reach downstream about 5 and 4.5 in the 2-D cylinder in Fig. 3, respectively. But in the perforated conic shroud in Fig. 6, contours of $\omega_{z}$ at -0.5 and -1 stretch downstream about 
6.1 and 5.9 at $z=0$, and 6.1 and 5.3 at $z=\lambda /(2 D)$, respectively. Owing to, at least, the strong streamwise interaction between $\omega_{x}$ and $\omega_{z}$, both the top and bottom shear layers are distinctly varied along the $z$-axis in a wave shape: staying apart from each other at the valley and close at the peak (see also in Fig. 6). Consequently, the wake width would be increased at the valley but reduced at the peak.

Now let us discuss two different models in describing the wavy shear layers. As reported in previous work of Darekar and Sherwin [14] for the flow around the square cylinder with wavy stagnation surfaces, the upper and lower shear layers were observed to be wavy across the span, as well as the near-wake width. The wake is wide behind the valley (the maximum point downstream) and narrow behind the peak (the maximum point upstream), as shown schematically in Fig. 29a. This three-dimensionality of the near wake is consistent with the appearance of the streamwise vorticity $\omega_{x}$ and the change in the vertical velocity $v$ distribution within the top and bottom shear layers. As shown in Fig. 29a, the streamwise vortex pair creates an upwash behind the peak and a downwash behind the valley, displacing the near wake in a sinusoidal fashion. The variation of $v$ within the shear layers will also contribute to the widening and narrowing of the near wake by pushing the free shear layers further apart in the vertical direction behind the valley where $v$ reaches maximum. This three-dimensionality of the shear layers is associated with the earlier separation of the shear layer just after the leading edge at the valley, while at the peak the shear layer remains attached as in the straight cylinder. This explanation is referenced as the model of the streamwise vorticity.

However, the streamwise vorticity model is still in question in explaining the following two cases: the П-type vortex appeared in the wake of the straight square cylinder at $R e=180$, and the far elongated shear layers with vortex shedding completely suppressed in sub-regime III-C in the wake of (peak-perforated) conic shroud at $R e=100$.

In the first case, it seems to have an obvious paradox if the streamwise vorticity model is used. As shown in Fig. 30a, the streamwise vorticity on the upper surface $A B$ is negative at $z=1.5$ and positive at $z=4.5$. This is supposed to result in the wake wide near $z=3$, but narrow near $z=0$. But, as shown in Fig. 30b, c, the wake width is obviously wide at $z=0$ but narrow at $z=3$. By carefully reviewing the distribution of streamwise vorticity in the shear layers and near wake, as shown in Fig. 16b, the sign of $\omega_{x}$ at $z=$ 1.5 is already changed to be positive early in the vortexformation region. This is apparently not generated from the upper surface. Then, if the model of the streamwise vorticity near the cylinder surface can be applied for the shear layers, the positive $\omega_{x}$ is just used to explain the wake narrowed at $z=3$. However, without the limitation of wall boundary condition, this explanation would be a little in trouble due to the adjacently downstream $\omega_{x}$ with the negative sign, greater spacial distribution and intensity, in the rolled-up lower shear layer.

In the second case, the model of the streamwise vorticity is not used enough to explain the wavy wake across the span. The streamwise vorticity just behind the body is decayed quickly downstream, as shown in Figs. 24 and 25. But the narrowed wake at the peak continues and is kept down to far away from the body. In the wake further downstream, only the vertical vorticity is existed and obviously greater than the streamwise vorticity in not only spacial distribution, but also intensity. This seems to indicate that the wavily varied wake may be also associated with the specific distribution of vertical vorticity.

Therefore, as reported in recent work by Lin et al. [22] for the flow past the harmonic and conic shrouds, an alternative model, called as the model of the vertical vorticity, was proposed. Based on the specific distribution of vertical vorticity $\omega_{y}$ in the shear layers, the relationship between the vertical vorticity and wavy wake is schematically shown in Fig. 30b. The existence of vertical vorticity changes the local distribution of streamwise velocity $u$ near the valley and peak. As shown in Fig. 30b, the reverse flow near the valley and downstream flow near the peak are induced by such vertical vortex pair. As a result, the streamwise velocity field would be redistributed to be increased at the peak and decreased at the valley. The wake profile, typically described by the streamwise velocity varied along the vertical direction $y$ at certain downstream position, is enlarged at the valley, but shrunk at the peak. Then the wake width, as well as the shear layers, is narrowed at the peak and widen at the valley in a sinusoidal fashion across the span too.

Now we have two models to explain the wavy shear layers and wake width across the span. The possibility of unifying these two models is naturally considered and discussed as follows, based on the analysis of vorticity components in the near wake.

\subsection{Spacial distribution of vorticity}

As for the spacial distribution of three components of vorticity, $\left(\omega_{x}, \omega_{y}, \omega_{z}\right)$, in the near wake of bluff body, there are three aspects that need to be inspected. The first is the sign of each component of vorticity. Here only the large-scale vortex, like the Kármán vortex, is taken into account. The second is the specific distribution of streamwise and vertical components of vorticity. The last is a combination of all three components of vorticity with specific signs in the near wake.

Let us start from the simplest case, only involving the spacial distribution of $\omega_{x}$ and $\omega_{y}$. As shown in Figs. 8 and 9 in sub-regime I-B, in region of $x>2$ and $z \in\left(0, \frac{1}{2} \frac{\lambda}{D}\right), \omega_{x}$ is always positive in the upper shear layer, but negative in the lower shear layer. While $\omega_{y}$ is always positive within these $\omega_{x}$ 


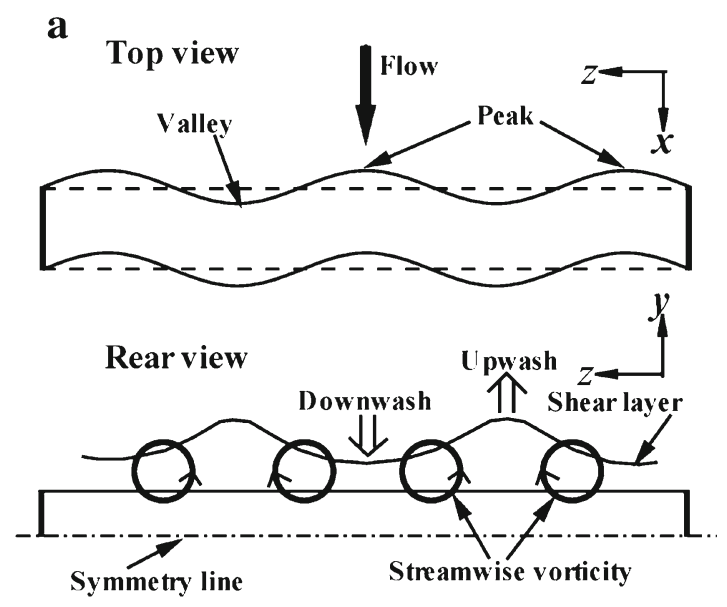

b

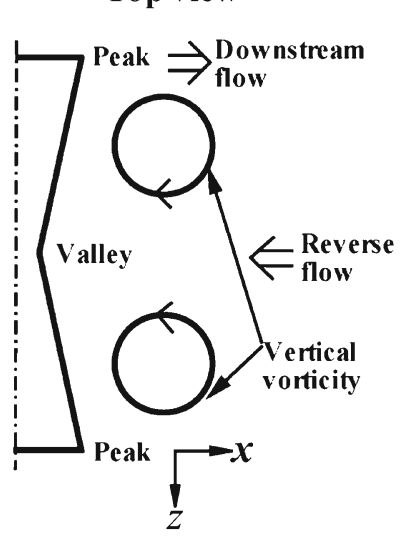

Side view

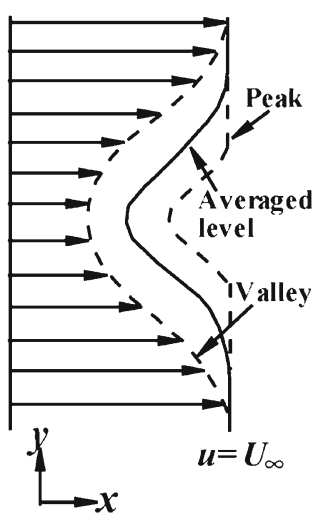

Fig. 29 Schematic views of the relation between a the streamwise vorticity or $\mathbf{b}$ the vertical vorticity and wake width variation along the span

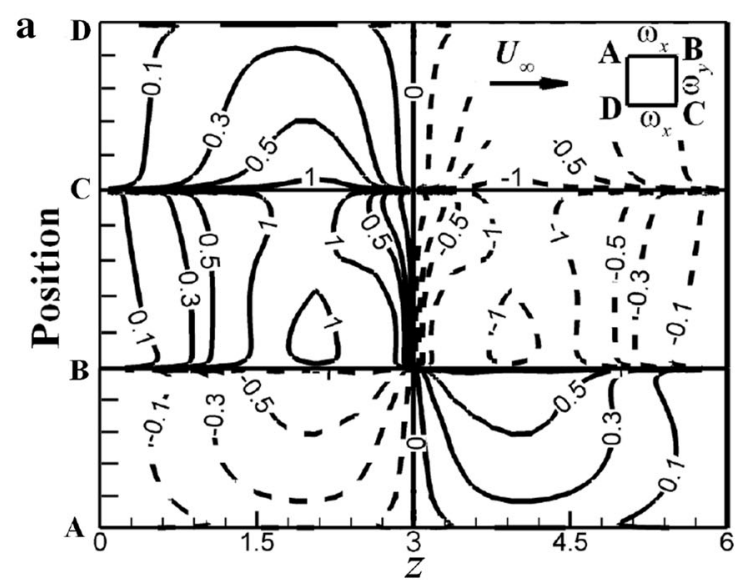

b
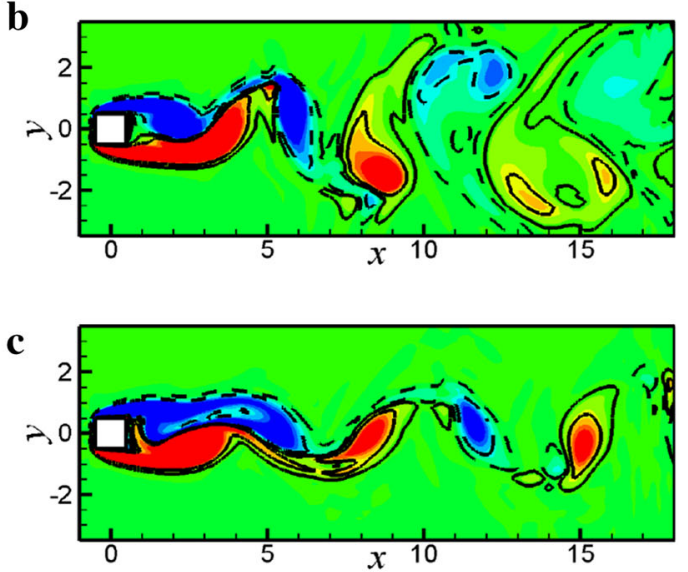

Fig. 30 Contours of $\mathbf{a} \omega_{x}(\mathrm{AB}$ and $\mathrm{CD})$ and $\omega_{y}$ (BC) on surfaces and $\omega_{z}$ at $\mathbf{b} z=0$ and $\mathbf{c} z=3$ in the near wake of the square cylinder at $t=340$ and $R e=180$ with non-dimensional spanwise computational length of 6 , where the red and blue colors, as well as the solid and dashed lines, denote the positive and negative values, respectively

emerged regions. And in region of $x>2$ and $z \in\left(\frac{1}{2} \frac{\lambda}{D}, \frac{\lambda}{D}\right)$, signs of $\omega_{x}$ and $\omega_{y}$ are distributed just oppositely to those in $z \in\left(0, \frac{1}{2} \frac{\lambda}{D}\right)$. As shown in Figs. 10-15, the similar sign law of $\omega_{x}$ and $\omega_{y}$ is also found out in region of $x>4-5$. Except these geometrically disturbed cylinders, the similar distribution of $\omega_{x}$ and $\omega_{y}$ with specific signs also appears in the wake of the straight square cylinder, as shown in Fig. 16 in the region of $x>3-4$.

This feature of $\omega_{x}$ and $\omega_{y}$ with specific signs provides an obvious evidence that two models of the streamwise and vertical components of vorticity are consistent with each other and unified. For example as shown in Figs. 6, 11, and 14, the narrowed wake width at the peak $\left(z=\frac{1}{2} \frac{\lambda}{D}\right)$ could be explained by either the model of the streamwise vorticity with positive sign or the model of the vertical vorticity with positive sign in the upper shear layer and spanwise region of $z \in\left(0, \frac{1}{2} \frac{\lambda}{D}\right)$.
Furthermore, let us review the streamwise and vertical interactions with the spanwise vorticity, as shown in Figs. 5 and 7. In Fig. 5, the streamwise vortex pair leads to the spanwise vortex distorted along the vertical and spanwise directions. From the point of view of the vortex line, the spanwise vortex without any distortion could be described by a straight vortex line across the span. After introducing the streamwise interaction, the spanwise vortex line is thus distorted and turned into downward $(-y)$ and upward $(+y)$. Accordingly, the vertical vorticity is generated. For example, the interaction between $+\left|\omega_{x}\right|$ and $-\left|\omega_{z}\right|$ leads to the appearance of $+\left|\omega_{y}\right|$, expressed by $\left(+\left|\omega_{x}\right|,+\left|\omega_{y}\right|,-\left|\omega_{z}\right|\right)$. While as for the interaction between $-\left|\omega_{x}\right|$ and $-\left|\omega_{z}\right|$, there is $\left(-\left|\omega_{x}\right|,-\left|\omega_{y}\right|,-\left|\omega_{z}\right|\right)$. Similarly for the vertical interaction in Fig. 7 for a simple case and schematically shown in Figs. 21 and 23 for more complicated vortex-shedding patterns, these two groups of sign combination for three components of vorticity are also existed. 
This analysis thereby leads to the conclusion that the streamwise and vertical interactions with the spanwise vortices, as well as two models of the streamwise and vertical components of vorticity, are really unified and can be used to explain the wavy shear layers, distorted spanwise vortex, and varied wake width across the span.

In addition, if the lower shear layer is considered, the following two groups of sign combination for three components of vorticity, according to their spacial distributions, are also obtained: $\left(+\left|\omega_{x}\right|,-\left|\omega_{y}\right|,+\left|\omega_{z}\right|\right)$ and $\left(-\left|\omega_{x}\right|,+\left|\omega_{y}\right|,+\left|\omega_{z}\right|\right)$.

\subsection{Sign laws of vorticity in the near wake}

Based on these four groups of sign combinations for three components of vorticity in the shear layers and near wake of bluff bodies, there are two sign laws obtained.

Only in the top or bottom shear layer is the first sign law obtained by combining streamwise and vertical components of vorticity. For instance, across the span, $\omega_{x} \cdot \omega_{y}$ is always positive in the top shear layer, but negative in the bottom shear layer, written by

$\operatorname{sgn}\left(\omega_{x} \cdot \omega_{y}\right)= \begin{cases}-1, & \text { bottom shear layer, } \\ 1, & \text { top shear layer, }\end{cases}$

where sgn is the sign function. Generally, the spanwise vortex with a negative sign is formed in the upper shear layer and shed, while the spanwise vortex with a positive sign is thus formed in the lower shear layer and shed alternatively.

Consequently, if all three components of vorticity in the near wake are included, the second sign law is obtained. For example, across the span, $\omega_{x} \cdot \omega_{y} \cdot \omega_{z}$ is always negative in the top shear layer or upper side of near wake ( $+y$ direction), and also negative in the bottom shear layer or down side of near wake ( $-y$ direction). Therefore, this sign law is also referred to as the negative sign law in the near wake of a bluff body, expressed by

$\operatorname{sgn}\left(\omega_{x} \cdot \omega_{y} \cdot \omega_{z}\right)=-1$

\section{Conclusions}

Based on flows around conic shrouds with and without peak perforations at $R e=100$ through numerical simulations, the focus is mainly put on the physical mechanisms in original spanwise vortex wavily distorted due to the introduction of 3-D geometric disturbances. The analysis is performed from the following several aspects: establishment of streamwise and vertical interactions with spanwise vortices, reexamination of vortex-shedding patterns in the near wake from the point of view of streamwise and vertical interactions, for- mation of wavy shear layers through two models and spacial distribution of three vorticity components with specific signs. Finally two sign laws are summarized.

The physical mechanisms responsible for the spanwise vortices distorted wavily across the span are attributed to two types of interaction with spanwise vortices in the near wake. The first is the streamwise interaction between streamwise and spanwise vortices, investigated in previous work [23], used to explain the spanwise vortices wavily curved along the vertical direction in the $(y, z)$ plane, while the second is the vertical interaction between vertical and spanwise vortices, proposed in present paper, suitable to illustrate the distorted spanwise vortices in the $(x, z)$ plane.

With the help of these two types of interaction, some vortex-shedding patterns appearing in different flow regimes can be properly illustrated, especially for their formation process. In the flow regime I, these two interactions are all weak due to weak disturbances and resultant weak streamwise and vertical vortices. The wake is, therefore, mainly described by the little distorted Kármán vortices alternatively shed. Even in regime II, although streamwise and vertical interactions are gradually enhanced, the obviously distorted spanwise vortices are still alternatively shed almost in synchronism along the spanwise direction. However, once disturbances are strong enough, vortex-shedding patterns in the near wake in regime III become more complicated. In the present paper, four basic effects due to the strong vertical interaction are identified and correlated with the $\Omega$-type vortex shed at the valley or peak, $\Omega$-type vortices shed alternatively at the valley and peak, and the complete suppression of Kármán vortices. Moreover, two vortex-shedding patterns in sub-regime III-D are analyzed and attributed to the dominantly strong streamwise interaction and coupled effects of strong streamwise and vertical interactions, respectively.

An interesting phenomenon, the particular distribution of streamwise and vertical components of vorticity with specific signs in the shear layers and near wake, is analyzed. Through the analysis of physical mechanisms of wavy shear layers and wake width across the span, two models of the streamwise and vertical components of vorticity are introduced and turned out to be unified. Similarly, the streamwise and vertical interactions with spanwise vortices are also proved to be unified based on spacial distributions of three components of vorticity in the near wake. Then two sign laws are obtained: the first sign law for $\omega_{x} \cdot \omega_{y}$ be positive in the upper shear layer but negative in the lower shear layer, and the second sign law for $\omega_{x} \cdot \omega_{y} \cdot \omega_{z}$ be always negative in the near wake.

In a future study, as a fundamental physical study, the theory of vortex-induced vortex (VIVor) would be proposed and used to explain the first and second sign laws in the near wake of any bluff body. And moreover, it is also interested whether these sign laws would be established in an internal flow through a pipe. 
Acknowledgements The project was financially supported by the National Key Scientific Instrument and Equipment Development Program of China (2011YQ120048).

\section{References}

1. Sarpkaya, T.: A critical review of the intrinsic nature of vortexinduced vibrations. J. Fluids Struct. 19, 389-447 (2004)

2. Williamson, C.H.K., Govardhan, R.: Vortex-induced vibrations. Annu. Rev. Fluid Mech. 36, 413-455 (2004)

3. Williamson, C.H.K., Govardhan, R.: A brief review of recent results in vortex-induced vibrations. J. Wind Eng. Ind. Aerodyn. 96, 713735 (2008)

4. Sarpkaya, T., Isaacson, M.: Mechanics of Wave Forces on Offshore Structures. Van Nostrand Reinhold Company, Washington (1981)

5. Kumar, R.A., Sohn, C.H., Gowda, B.H.L.: Passive control of vortex-induced vibrations: an overview. Recent Patents Mech. Eng. 1, 1-11 (2008)

6. Owe, J.C., Bearman, P.W., Szewczyk, A.A.: Passive control of VIV with drag reduction. J. Fluid Struct. 15, 597-605 (2001)

7. Korkischko, I., Meneghini, J.R.: Suppression of vortex-induced vibration using moving surface boundary-layer control. J. Fluids Struct. 34, 259-270 (2012)

8. King, R., Brown, A., Braaten, H., et al.: Suppressing full-scale riser VIV with the VT suppressor. In: Proceedings of 32nd International Conference on Ocean, Offshore and Arctic Engineering (OMAE2013) (ASME), Nantes, France, June 9-14, OMAE2013$11642(2013)$

9. Xu, F., Chen, W.L., Xiao, Y.Q., et al.: Numerical study on the suppression of the vortex-induced vibration of an elastically mounted cylinder by a travelling wave wall. J. Fluids Struct. 44, 145-165 (2014)

10. Lee, L., Allen, D.W.: The dynamic stability of short fairings. In: Offshore Technical Conference, Houston, TX, USA, OTC-17125 (2005)

11. Huera-Huarte, F.J.: On splitter plate coverage for suppression of vortex-induced vibrations of flexible cylinders. Appl. Ocean Res. 48, 244-249 (2014)
12. Trim, A.D., Braaten, H., Lie, H., et al.: Experimental investigation of vortex-induced vibration of long marine risers. J. Fluid Struct. 21, 335-361 (2005)

13. Owen, J.C., Szewczyk, A.A., Bearman, P.W.: Suppression of Kármán vortex shedding. Gallery Fluid Motion Phys. Fluids 12, 1-13 (2000)

14. Darekar, R.M., Sherwin, S.J.: Flow past a square-section cylinder with a wavy stagnation face. J. Fluid Mech. 426, 263-295 (2001)

15. Lam, K., Lin, Y.F.: Effects of wavelength and amplitude of a wavy cylinder in cross-flow at low Reynolds numbers. J. Fluid Mech. 620, 195-220 (2009)

16. Lin, L.M., Zhong, X.F., Wu, Y.X.: Flow around a circular cylinder with radial disturbances at a low Reynolds number. In: Proceedings of Twenty-third International Offshore Polar Engineering Conference (ISOPE), Anchorage, Alaska, USA, June 30-July 5, 387-394 (2013)

17. Lin, L.M., Zhong, X.F., Wu, Y.X.: Effect of peak perforation on flow past a conic cylinder at $R e=100$ : drag, lift and Strouhal number. China Ocean Eng. 31, 330-340 (2017)

18. Roshko, A.: On the Development of Turbulent Wakes from Vortex Streets. NACA Report 1191 (1954)

19. Williamson, C.H.K.: Vortex dynamics in the cylinder wake. Annu. Rev. Fluid Mech. 28, 477-539 (1996)

20. Lin, L.M.: Wake dynamics and forces in the flow around the square-section cylinder with a geometric disturbance. Ph.D. Thesis, Institute of Mechanics, CAS, Beijing, China (2007) (in Chinese)

21. Lin, L.M., Zhong, X.F., Wu, Y.X.: Effect of perforation on flow past a conic cylinder at $R e=100$ : vortex shedding pattern and force history. Acta Mech. Sin. 34, 238-256 (2018)

22. Lin, L.M., Zhong, X.F., Wu, Y.X.: Characteristics for a flow past a circular cylinder with two types of radial disturbances at $R e=100$. Adv. Mater. Res. 871, 107-114 (2014)

23. Meiburg, E., Lasheras, J.C.: Experimental and numerical investigation of the three-dimensional transition in plane wakes. J. Fluid Mech. 190, 1-37 (1988)

24. Henderson, R.D.: Nonlinear dynamics and pattern formation in turbulent wake transition. J. Fluid Mech. 352, 65-112 (1997)

25. Newman, D.J., Karniadakis, G.E.: A direct numerical simulation study of flow past a freely vibrating cable. J. Fluid Mech. 344, 95-136 (1997) 